\title{
How to Face Skin Cancer with Nanomaterials: A Review
}

\author{
Fakhara Sabir ${ }^{1}$, Mahmood Barani ${ }^{2}$ (D), Abbas Rahdar ${ }^{3,}$ *, Muhammad Bilal 4(D), Muhammad Nadeem \\ Zafar $^{5(D)}$, Simona Bungau ${ }^{(1 D}$, George Z. Kyzas ${ }^{7, *(D)}$
}

1 University of Szeged, Faculty of Pharmacy, Institute of Pharmaceutical Technology and Regulatory Affairs, Szeged, H6720, Hungary; fakhra.sabir@gmail.com (F.S.);

2 Department of Chemistry, Shahid Bahonar University of Kerman, Kerman 76169-14111, Iran; mahmoodbarani7@gmail.com (M.B.);

3 Department of Physics, University of Zabol, Zabol 98613-35856, Iran; a.rahdar@uoz.ac.ir (A.R.);

4 School of Life Science and Food Engineering, Huaiyin Institute of Technology, Huaian 223003, China; bilaluaf@hotmail.com (M.B.);

5 Department of Chemistry, University of Gujrat, Gujrat 50700, Pakistan; znadeempk@ gmail.com (M.N.Z.);

6 Department of Pharmacy, Faculty of Medicine and Pharmacy, University of Oradea, Oradea 410028, Romania; sbungau@uoradea.ro (S.B.);

7 Department of Chemistry, International Hellenic University, Kavala 65404, Greece; kyzas@ chem.ihu.gr (G.Z.K.);

* Correspondencekyzas@chem.ihu.gr (G.Z.K.); a.rahdar@uoz.ac.ir (A.R.);

Received: 25.11.2020; Revised: 16.12.2020; Accepted: 17.12.2020; Published: 21.12.2020

\begin{abstract}
Nanotechnology is one of the most attractive disciplines ranging from materials science to biomedicine. It is one of the most prominent areas of research for targeting skin melanoma and other diseases. The application of nanomaterials in the field of skin has increased interest in topical targeting to increase the permeation and retention and reduce its side effects. Different types of nanomaterials have been used for the application of skin diseases and cancer. These nanomaterials include carbonnanotubes, liposomes, nanoemulsion, nano micelles, etc. In the United States and globally, the prevalence of melanoma has had a growing rise throughout the last 50 years. This review seeks to discuss various types of treatment and diagnosis of early-stage skin cancer. There are many skin cancer biomarkers for early detection of this type of cancer. Diagnosis of skin cancer forms a way of traditional approaches, and their weaknesses are discussed. Electrochemical and optical biosensors for the diagnosis and monitoring of skin cancer are also was described. After all, some issues should be addressed and overcome regarding the competition of new techniques in cost and convenience of use.
\end{abstract}

Keywords: nanomaterials; skin; cancer; nanoparticles; diagnosis; treatment.

(C) 2020 by the authors. This article is an open-access article distributed under the terms and conditions of the Creative Commons Attribution (CC BY) license (https://creativecommons.org/licenses/by/4.0/).

\section{Introduction}

Skin cancer is a broad term that describes a large number of different malignant lesions of the skin. It can be further categorized into two most common tumors: keratinocyte cancer (also recognized as non-melanoma skin cancer) and melanoma skin cancer. Among these, nonmelanoma skin cancer is a widely detected type of cancer globally. In contrast, melanoma skin cancer results in the highest percentage of deaths [1]. The skin's rarer tumors, like cutaneous lymphoma, Kaposi sarcoma, and Merkel Cell Carcinoma, have also been described [2]. Recent epidemiological data revealed the diagnosis of over 5.4 million cases of skin cancer in the United States in 2012 [3]. In the U.S., skin cancer frequency has dramatically increased (>300\%) between 1994 and 2014. In 2018, the highest incidence of skin cancer was reported in Australia, affecting approximately 300/100,000 people [4]. Therefore, skin cancer 
characterizes an extensive global health burden with huge psychosocial effects and necessitates significant attention in terms of technologies and treatments.

Various choices, including surgery, radiotherapy, and chemotherapy, are available to treat skin cancer. Surgical procedures for treating skin cancers include simple excision of tumor cells from the skin, shave-excision (abnormal skin area is removed with a small blade), Mohs surgery (excision of a thin layer of skin from the tumor region followed by viewing under a microscope to inspect the presence/absence of tumor cells), cryosurgery (freezing of localized affected tissue and excision by surgery), and curettage and desiccation (treatment to remove affected skin surface with a curette) [5]. Radiation therapy can be classified into conventional external radiation therapy, superficial x-ray therapy, and brachytherapy. Volumetric modulated arc therapy is the latest and novel radiation modality that ensures the continuous delivery of radiation dose to targeted tumor tissue while circumventing or minimizing the dose to healthy tissue around the tumor microenvironment [6]. Nevertheless, the lack of histological clearance, the evolution of undesirable phenotypes in recurrent tumors, and elevated costs are significant drawbacks associated with the radiation technique [7]. Chemotherapy implicates the use of potent chemicals in the body to destroy cells proliferated in an unprecedented way. This method is most widely applied for cancer treatment because the tumor cells multiply and grow faster than normal body cells. Numerous chemotherapy drugs, including imiquimod, carboplatin, 5fluorouracil, sonidegib, trametinib, vemurafenib, vismodegib, dabrafenib, temozolomide, cobimetinib, dacarbazine, imatinib, binimetinib, selumetinib, nilotinib, nitrourea, and taxanes are available for the treatment of skin cancer. The drugs contain both topical and can be administered orally in the form of capsules or tablets, which exert a widespread systemic effect due to distribution in various body parts via the bloodstream. However, these remedies are not effective in the complete abatement of cancer. They are associated with many adverse impacts causing discomfort to the patients. The lack of specificity to cancerous cells often results in damaging effects on the healthy cells [5].

Given current advances in cancer therapy, nanosystems' application has gained incredible interest as a promising approach for transporting therapeutic molecules and drugs $[8,9]$; [10]. These nanosystems can be designed for overcoming biological barriers and targeted delivery of chemotherapeutic drugs to the tumor microenvironment, thus permitting the use of minimum doses, improving treatment efficiency, and circumventing the extent of side effects [11, 12]; [13]; [8]. Nanoparticles as drug carriers provide impressive developments in skin cancer's therapeutic treatment by effective delivery, bioavailability, enhanced cellular specificity, ability to endure against multi-drug resistance, and improved therapeutic efficiency [14-16]. In addition, nanoparticle-driven drug transport can increase drug retention along with tunable release at the targeted affected region inside the skin. The nanoparticulate system has been employed in many applications, such as drug delivery, tumor targeting, cell imaging, and image-directed tumor ablation [17-19]. Nanoparticles can penetrate through the skin, reaching the specific tumor site for directed drug delivery by surface modification. In recent years, extensive research has been executed to develop useful and novel nanoparticles with promising drug delivery efficiency to treat skin cancer. Despite the apparent advantages of nanotherapeutics for cancer treatment, most nanotechnology-based approaches are at the development stage. However, some of the nanoformulations have entered clinical practice and already available in the market. For example, doxorubicin containing PEGylated liposome, Doxil@, is being used for ovarian and breast cancer, Kaposi sarcoma, and multiple myeloma [13]. Abraxane $®$, albumin-bound paclitaxel nanoparticles, is another nanoformulation that has 
been approved by the FDA in 2005. It is used to treat various cancers, particularly non-smallcell lung cancer and metastatic breast carcinoma [11, 12].

Regarding skin cancer, a large number of nanostructured materials, including nanotubes, quantum dots, liposomes, dendrimers, nano micelles, polymersomes, gold NPs, nanogels, silica nanoparticles, polymeric nanoparticles, nanospheres, magnetic NPs, nanostructured lipid carriers, and solid lipid nanoparticles have been successfully fabricated and characterized for treating brain cancers proficiently [5]. These nanomodalities have also been enormously involved in detecting skin cancer with superior sensitivity, cost-effectiveness, minimal underdiagnosis, and overdiagnosis of cancer [20]. In this paper, we studied the recent reports on applying various nanomaterials to treat and diagnose skin cancer. Therefore, our review spotlights an up-to-date and comprehensive outlook of the various types of nanomaterials that have been tested in the delivery of drugs for skin cancers - the information presented to attract the attention of all those interested in the NPs-skin cancer association.

So with this in mind, in continuation of our group's efforts related to the synthesis of nanomaterials and investigation about their potential bioapplications [9, 20-47], in the current work, we reviewed different nanomaterials applied to diagnosis and treatment of skin cancer.

\section{Nanomaterials for Targeting Skin Melanoma (Cancer)}

The treatment of skin diseases is the most feasible approach compared to the other invasive routes like parenteral or oral drug administration. Nanosized particles increase focus for skin targeting for diseases related to the skin to enhance compounds' permeation to reduce their adverse reactions. Several different types of nanomaterials are understudy for the treatment of skin diseases and melanoma. These include dendrimers, liposomes, carbon derived nanoparticles, protein-based, and inorganic nanoformulation. Applications of drugs or active pharmaceutical ingredients (API) to the skin surface avoid major shifts in the plasma levels typical for the frequent intervention of fast eliminating drug compounds. While it also allows API to avoid the first-pass metabolism through the liver after absorption into the intestine. Transdermal drug delivery route has gained significant importance for systemic treatment, e.g., by using drugs such as estrogen or glyceryl trinitrate for chronic suppression of pain [48, 49].

The skin application of various drugs cannot target the API for skin melanoma and several other diseases and for anti-rheumatic therapy to avoid nonsteroidal anti-inflammatory drug's side effects. Researchers are also making significant contributions over the last decades to kill the malignant cells through specific targeting. Nanodermatology has been applied for both the treatment and diagnosis of skin cancer. The implementation of nanoparticles to provide targeted delivery in skin melanoma is widely expected to change cancer therapeutics' landscape in the foreseeable future. These nanoparticles can bind the polymer into a malignancy cell membrane or to cytoplasmic or nuclear receptor sites to enhance the concentration at the target site and reduce its toxicity. The skin application mode has been used by physicians, not only by dermatologists, that are trying to quantify the amount of API circulates within the entire organism. The drug carriers responsible for overcoming the stratum corneum's barrier feature are the main issue of current research [50-52].

\subsection{Lipid nanoparticles.}

Lipid nanoparticles are among the most biocompatible form of nanoparticles studied for the skin. Nanoemulsion, liposomes, solid lipid nanoparticles, nanostructured lipid cargos 
are major types of nanoparticles evaluated in skin permeation studies. The other common types of lipid nanoformulation used for skin delivery, either topical or transdermal delivery, mentioned in literature are cubosomes, nanodispersion, and niosomes. These drug delivery systems have been used because of their advantageous properties like occlusive properties, alteration in release pattern, and enhanced skin perforation linked with fewer side effects[53]. One new type of liposome called elastic type can modulate the capillaries flow via tiny pores of the skin. It is stated that this lipid formulation can penetrate and take the compound into deep down layers of the skin. For acute and chronic dermatitis treatment, liposomes encapsulated with dipotassium glycyrrhizinate have been used as an anti-inflammatory agent to target the disease. In the dermatological field, liposomes were used as a lubricating and moisturizing agent. Active substances such as retinoids, glucocorticosteroids encapsulated into liposomes showed enhanced efficacy and decreased their side effects. The topical liposomes are superior to conventional ones in some skin diseases such as atopic eczema [54]. Figure 1 showed a list of nanomaterials for skin diseases and skin cancer.

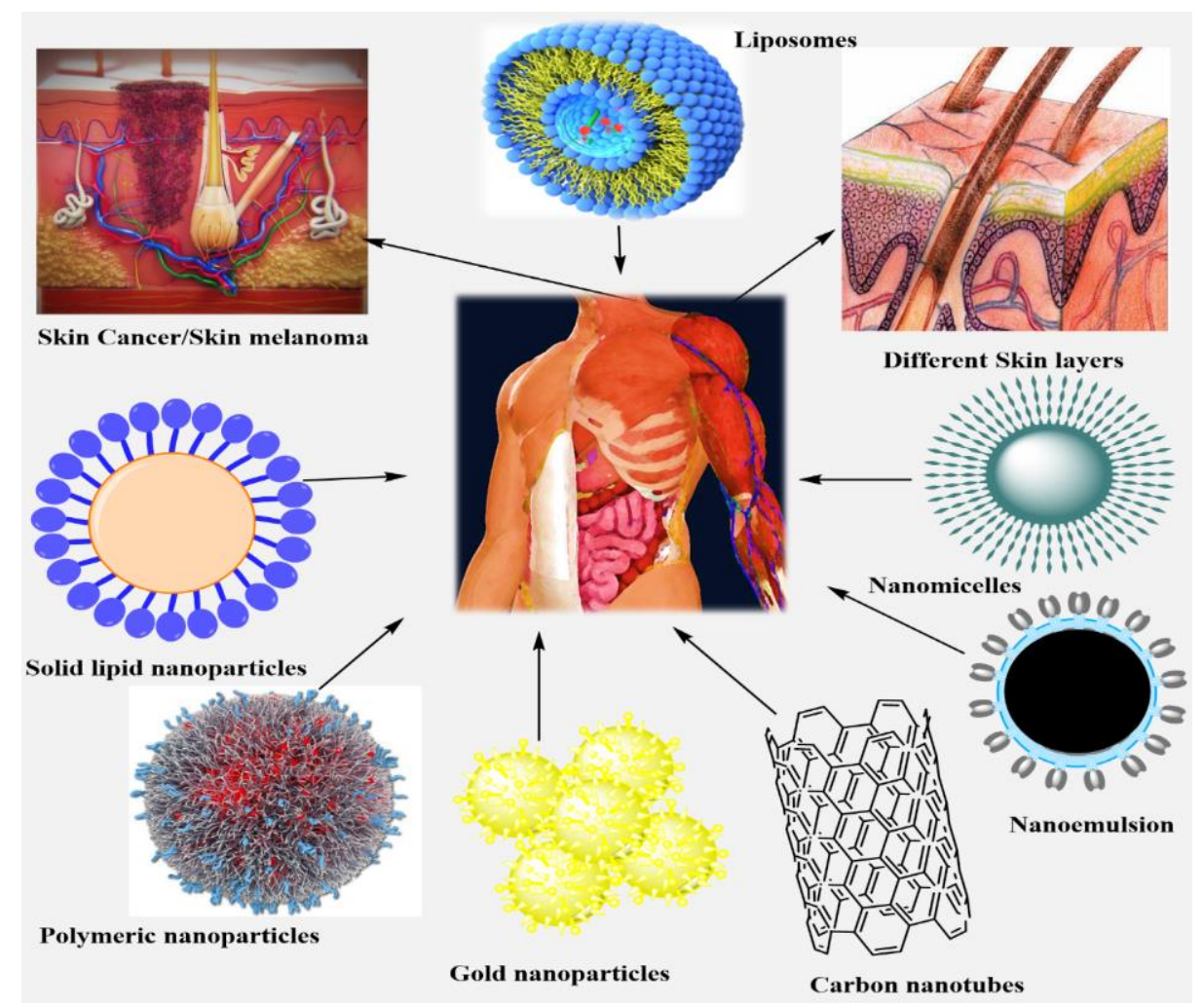

Figure 1. Application of Nanodermatology in treatment of skin diseases and melanoma.

Jain et al. define enhanced delivery of minoxidil encapsulated into neutral liposomes into hair follicles compared to other formulations. The other drugs like finasteride for treating alopecia androgenetica treatment. Anti-androgens, including RU 68841 myristate encapsulated solid lipid nanoparticles reported to enhance the permeation and delivery of the active pharmaceutical compounds. The liposomal formulations of cyclosporine A stimulated the reproduction of hairs in rats and demonstrating a significant approach for topical administration of alopecia areata in humans [55]. The topically applied solid lipid nanoparticles (SLNs) were considered to lose the skin pores structure. This modification can decrease the skin barrier attribute and can occlude the skin surface. The topically delivered hyaluronic acid comprising nano-sized particles can easily permeate via the skin surface. Nano-structured lipid carrier encapsulated with an active pharmaceutical ingredient for sustained release [56]. 
Anup Jose et al. studied the effectiveness of co-encapsulated curcumin and anti-STAT3 siRNA using cationic charged liposomes against skin melanoma. The liposomes were characterized for measuring the encapsulation efficiency, zeta potential, particle size. The cell line studies on B16F10 viability studies on mouse melanoma cells showed that both drugs' coencapsulation inhibited the cancer cell growth compared to other formulations. The positive liposomes loaded with curcumin could breach the skin up to specified depth after using the iontophoretic technique. The in vivo studies were conducted in the melanoma skin cancer cell model in mice. The loading of curcumin and genetic material (STAT3 siRNA) by utilizing the liposomes stops tumor proliferation as calculated by tumor weight and volume with either curcumin or liposomal STAT3 siRNA.

Moreover, the curcumin encapsulated liposomes-siRNA complex demonstrates the effectiveness parallel to inhibiting the tumor proliferation and STAT3 protein compared to intervention intratumorally given. It is concluded that positively charged liposomes can be applied topically via iontophoretic co-encapsulation of small molecule and siRNA to efficiently treat skin diseases [57]. Carla Caddeo et al. investigated the fabrication of liposomes for the co-administration of natural polyphenols, including resveratrol and quercetin. The polyphenols, when co-loaded into liposomes, demonstrated higher cellular uptake rather than a single agent. This study resulted in a higher capability to scavenge ROS in fibroblast. The efficacy of polyphenols loaded into liposomes was measured in a mouse model of skin eruption.

The liposomal topical administration leads to a tremendous improvement in tissue impairment by reducing leukocyte infiltration and edema. Hence, the following study concluded that the polyphenol encapsulation into liposomes helps treat oxidative stress or inflammation linked with malignant skin lesions [58]. Raquek Petrilli et al., the SCC (squamous cell carcinoma) is a type of tumor in which epidermal growth factor receptor is highly expressed because of malignancy. For the treatment of SCC, cetuximab (an anti-EGFR antibody) is administrated in addition to the chemotherapeutic drug for enhanced efficacy. In this work, the EGRF targeted immuno-liposomes encapsulated with 5-fluorouracil (5-FU) were developed to target the SCC cells. The subcutaneous injections were topically applied. For 5FU-loaded immunoliposomes, iontophoresis was employed in the SCC xenograft animal model to analyze the intervention route's influence on therapeutic efficacy. The cellular uptake studies of immunoliposomes loaded with cetuximab by EGFR positive SCC cells was 3.5 times greater than the uptake of liposomes given to the control group. The skin permeation studies results showed that immune liposomes iontophoresis twice the 5-FU permeation into the epidermis compared to the same treatment with control liposomes administered to the control group. In the in vivo studies, the subcutaneous injection of immune-liposomes decreases the tumor volume up to or greater than $60 \%$ compared to the negative control. The $50 \%$ decrease in tumor volume compared with control and 5-FU solution that control liposomes treatment. This study concluded that the immuno-liposomes encapsulated 5-FU topical administration using iontophoresis is a more convenient approach for SCC targeted treatment[59].

\subsection{Polymeric nanoparticles.}

Polymeric nanoparticles have great importance in skin application due to their greater stability and controlled release and permeation via polymeric matrix to permeate the skin. These polymeric nanoparticles maintain the design for an extended period of time [60]. For example, hydrogel encapsulated dexamethasone has greater efficacy to be applied to treat 
psoriasis as a controlled delivery product [61]. Polymeric nanoparticles encapsulated with siRNA (small inhibitor ribonucleic acid) may specifically inactivate the gene expression. Nano-encapsulation of siRNA has been applied to treat the pachyonychia congenital and targeted delivery to inhibit a test gene expression in melanoma in human trials. Polymeric Nano-capsules are NPs smaller than $1 \mu \mathrm{m}$ comprised of lipid core and covered with ultrathin polymeric outer surface stabilized by surfactant.

Thermo-sensitive polymers load the drugs solubilize to release along with having a low value of critical temperature. These are being applied for drug delivery at the targeted delivery where external heat stimulus is applied or at the site of inflammation [60]. The application of methotrexate-loaded thermo-sensitive polymer was used as a basis for the treatment of psoriasis. The pharmaceutical use of proteins and peptides elaborated in the present years includes autoimmune disease, infection, and other anti-aging treatments. The encapsulation of hair growth compounds in PLGA nanoparticles will be enhanced their permeation within hair follicles up to two times in the case of the control solutions. The loading of minoxidil in poly(ecaprolactone) block polyethylene glycol NPs enhanced the hair follicles' permeation [62].

The natural polymeric NPs, like chitosan, gelatin, albumin, alginate, are most frequently is used for topical skin delivery and targeting skin melanoma.

The chitosan, the de-acetylated derivative of chitin, is a biodegradable polymer comprises mainly of glucosamine units. The anti-microbial, anti-oxidant, and anti-inflammatory properties make chitosan a suitable carrier for targeting therapeutics to treat skin disorders. However, at normal $\mathrm{pH}$, the amine groups of chitosan are protonated, and therefore chitosan is positively charged. The cationic charge could be used for NPs in solution through crosslinking with polyanions to more efficaciously incorporate the anionic drugs via electrostatic interaction and promote the cellular internalization of drug encapsulated chitosan NPs [63].

Hasanovic et al. showed that the encapsulation of acyclovir (anti-viral drug into chitosan-TPP) NPs resulted in enhanced drug permeation, low photo-degradation, increase drug permeation via porcine skin [64]. Ozbas Turan et al. demonstrated that the antisense oligonucleotide-loaded chitosan NPs at a dose of 15-90 $\mu \mathrm{g}$ showed greater inhibition of $\beta$-gal expression after 6 days post-transfection in rats [65]. Marina Franca Dias et al., the antineoplastic agent imiquimod (Imq) is utilized to treat skin cancer. However, the greater number of local and systemic adverse effects related to the use of less skin penetration could impair the therapeutic efficacy. The following study aims to evaluate the anti-angiogenic effect and antitumoral efficacy of polymeric nanoparticles encapsulated with Imq. The anti-angiogenic efficacy of the fabricated formulation was evaluated in chorioallantoic embryo in chicken. Also, its chemopreventive potential was evaluated in the carcinogenesis model in mice. It concluded that the formulated delivery system could be used as an alternative route to treat the diseases relevant to the vessel formation and enhance skin efficacy and penetration of less soluble drugs to target skin diseases, including skin melanoma [66].

Gamal-Eldeen et al. worked to encapsulate the indocyanine green (ICG) dye in polymeric nanoparticles to enhance loaded ICG components' aqueous stability. This study evaluated the PDT effectiveness of anti-EGFR conjugate and free ICG-PEEBBLE. The findings of the following study clearly showed that the COX-2 (cyclooxygenase-2), TNF- $\alpha$ (tumor necrosis factor), 5-LOX (5-lipoxygenase) were decreased. And the apoptosis, caspase3 , and histone acetylation were generated in transgenic mice after PDT using both types of formulation. It is concluded for the following study that conjugation of indocyanine green- 
PEBBLE to anti-EGFR was very efficient in blocking the VEGF and also enhance the caspase3 in comparison to free ICG-PEBBLE [67].

Pandey et al. studied atopic dermatitis while fabricating the chitosan nanoparticles for dermal targeting and increasing the subcutaneous tissue. The developed nanoparticles were investigated for polydispersity index (PDI), zeta potential, particle size, morphology, etc. The results indicated that the optimized formulation showed optimum results. In vitro release study revealed that release under simulated skin conditions. Permeation study of BMV (Betamethasone valerate) was more significant via BMV-CS-NPs; however, the quantity of drug into epidermis and dermis was greater HA-BMVCS-NPs, when compared to BMV-CSNPs. It is stated that HABMV-CS is an efficient delivery system for more targeted delivery and enhanced anti-AD efficacy[68]. Figure 2 describes the penetration of CS-BMV-NPs for the treatment of atopic dermatitis.

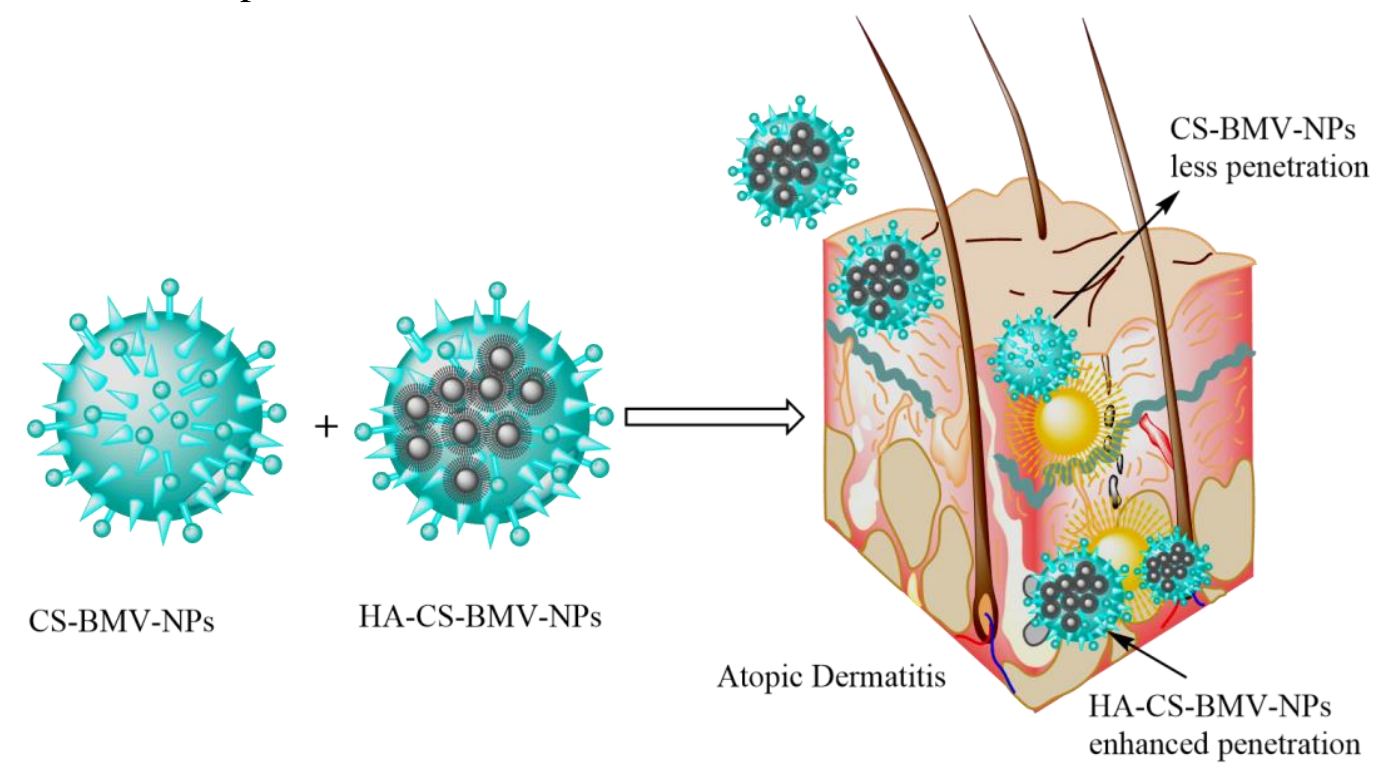

Figure 2. A comparative study of atopic dermatitis treatment through hyaluronic acid-coated BMV encapsulated CS-nanoparticles and without hyaluronic acid coating CS-BMV-NPs.

Bhatnagar et al. developed the bromelain encapsulated poly (lactic-co-glycolic acid) and studied the anti-tumor effects in the skin tumorigenesis mice model. The following study results revealed the increased anti-neoplastic effect of NPs in skin cancer model stage II. The decline in the number of the tumor cells, a decrease in percent tumorigenesis, and percent mortality in mice were seen in NPs loaded BL compared to free BL. These effects were also supported by histopathological evaluations. The results demonstrated that the NPs were fabricated to enhance chemotherapy's efficiency against skin melanoma at small doses[69]. Samaneh Bayat et al. studied the bromelain encapsulated chitosan nanofibers for burn injuries in laboratory animal models. Chitosan nanofibers loaded with bromelain developed by electrospinning technique. The physiochemical characteristics of nanofibers were analyzed. Cytotoxicity analysis was also performed using Alamar blue. The healing process of chitosan nanofibers was studied in rats for 21 days. The study analysis showed that $2 \%$ chitosan w/v bromelain encapsulated nanofiber was efficient to repair the skin burn [70].

\subsection{Nanoemulsions.}

Nanoemulsions are stable systems with a clear appearance and carrying special rheological characteristics. It is a most used delivery system for the sustained release of APIs 
into a deep layer of skin. The nano-emulsion advantage over others is that it can decrease transepidermal water loss (TEWL) by hydrating the skin and are more permeable to APIs. The greater solubilization and kinetic stability make them an attractive formulation.

Severino et al. worked on squamous cell carcinoma and basal cell carcinoma, which were reported to be among the most common malignancies. Chemotherapeutics used under similar conditions showed nonselective targeting along with severe side effects. These types of nanoparticles showed sustained release and stability of encapsulated drugs from chemical degradation. The following technique is very useful for increasing the intracellular quantity of drugs and decreasing the cytotoxicity [50].

Primo et al. worked on the development of a novel delivery system consisting of surfactants with magnetic fluid (obtained from maghemite) citrate derivative. The in vitro studies showed that the formulation presents a significant potential for synergetic application in the topical release of photosensitivity drugs and shown potential tissues targeting properties in the photodynamic therapy. In vitro studies revealed that tape stripping could be used to analyze drugs in the stratum corneum and dermis+epidermis skin layers. The following nanoemulsion were applied on the pigskin surface in the Franz diffusion cell. The results concluded that the magnetic nano-emulsion would enhance the drug release on the skin layers deep down compared to traditional formulation having no magnetic nanoparticles. This is all related to enhancing the biocompatibility of nanoformulation because of enhancing efficacy for the extracellular matrix in the skin and enhancing the drug-loaded inside the corneocytes wall [71]. Primo et al. developed the magnetic nanoemulsion to apply in magnetic hyperthermia and photodynamic treatment. It is concluded that after the intervention of a very little amount of drug, the tumor inhibition gets enhanced [72].

Giacone et al. studied aimed to fabricate and evaluate nano-emulsion for topical skin delivery of the cytotoxic agent piplartine. The cytotoxicity study was approximately 2.8 fold higher when the drug was loaded in chitosan nanoemulsion for targeting the melanoma cells. The efficacy of this nano-system on melanoma tissues was concentration relevant. The results supported the significant application of the chitosan modified nanoemulsion loaded with piplartine to manage skin cancer [73].

Falamas et al. proposed the study to fabricate the ex vivo and in vivo surface-enhanced signal from mice's skin and then used it against various skin pathologies, including skin cancer. The chemopreventive activity of natural compound betulin was studied in skin carcinoma in mice models. The study of various skin pathologies' differentiation was done by performing $\mathrm{K}$ means clustering and components analysis. The efficiency of nanoemulsion loaded with botulin validated by histological study and the chemometrics techniques showed the direct SERS differentiation link among the botulin and cancerous nanoemulsion treated skin [74].

Yousef et al. developed curcumin-loaded nanoemulsion against several skin diseases, including melanoma and psoriasis. To optimize skin delivery formulation, the curcumin $\mathrm{QbD}$ (Quality by Design) approach is utilized to enhance the skin targeting of curcumin. It is concluded that the efficacy of nanoemulsion for skin targeting of the curcumin, and showed the mechanism of enhanced permeation. The results concluded that curcumin loaded nanoemulsion has significant potential to target psoriasis and skin melanoma [75].

\subsection{Carbon nanotubes.}

The CNTs (carbon nanotubes) are the most stable compounds with good cytoprotective and anti-oxidant effects. The CNTs conductivity was applied to develop a very good biomarker 
sensor for skin melanoma diagnosis and infections at its early stage[76]. Anna Shvedova et al. evaluated and screened the effects of carbon nanotubes by utilizing immortalized keratinocytes' cell culture. The longer exposure of single-wall carbon nanotube (SWCNT), cellular toxicity, and oxidative stress were analyzed by loss of cell viability, free radicals formation, and aggregation of per-oxidative products. SWCNT exposure also results in morphological and ultra-structural modifications in cultured skin cells. It is concluded that skin exposure to unrefined SWCNT may take to skin toxicity because of accelerated oxidative stress in workers' skin [77].

Ding et al. use nanotechnology to evaluate the significance of toxic effects on the environment and people. To evaluate the complete genomic expression analysis and enhanced phenotypic measurement on human skin, the dermal fibroblast cell populations were exposed to MWCNTs. The results showed the exposing cells to both types of nanotubes at cytotoxic doses induced cell cycle arrest that enhances the necrosis. Moreover, these nanotubes' exposure stimulates the genes involved in cellular transport, metabolism and cell regulation, etc. Microarray analysis of the promotor analysis showed that p38/ERK-MAPK and interferon cascades are critical pathway components in the signal transduction, participating in the more adverse effects observed upon nanotubes exposure[78].

Hasebi et al., the development of stable anticancer drugs and CNTs has been under investigation. The nanotubes loaded with two drugs, aminolevulinic acid and tretinoin, evaluated for their stability of the composites. The results showed that tretinoin encapsulated nanotube is more stable than nanotube-aminolevulinic acid [79].

Moon et al. evaluated the photothermal therapy using nanomaterials as an efficacy technique for cancer targeting. SWNT is a potential and efficacious candidate as a photothermal agent. This study demonstrated in vivo obliteration of malignant tumors by targeting the SWNTs and NIR irradiation. The photo-thermally targeted mice showed a complete cure of tumor without recurrence phase for 6 months. Most of the SWNTs that intervene were removed out from the mice in about 2 months. The results concluded that SWNTs serve as an efficient photothermal agent and a step towards future cancer therapeutics [80].

Nanda Gopal Sahoo et al. fabricated multiwalled carbon nanotubes and graphene oxide functionalized by highly biocompatible and hydrophilic for loading and targeting an anticancer compound CPT camptothecin. The MWCNT-PVA and GO-PVA were encapsulated via pi-pi interactions and showed the potential to kill the breast and skin cancer cells [81].

\subsection{Nanomicelles.}

The nano-micelles have been used for drugs targeting the skin. These nano-carriers have few limitations in that they are only deposited in the hair follicles. They might not reach into the skin layer deep down $[82,83]$. Emine Kahraman et al. developed the nano-micelles loaded with terpenes to meet the challenges and evaluate skin delivery potential. The drug accumulation and permeation were evaluated in the skin by the tape stripping method. The data showed that nano-micelles loaded with terpinolene may cause the aggregation of more concentration of drug in the striped skin as compare to the commercial product and micelles without terpene. The loading of terpinolene into nanomicelles can be a more feasible approach for drug delivery [82].

Wang et al. develop and design nano-carriers from polymers for targeting the siRNA to skin melanoma for transdermal application. Encapsulated siRNA reproduces the efficacy in 
inhibiting the proliferation of skin cancer. The study showed that cationic micelles are effective as gene delivery nanoplatforms for melanoma therapy [84].

Yang et al. developed in situ gel for controlled drug delivery, encapsulated with curcumin-loaded micelles, and suitable for skin repair. Studies suggested that curcumin loaded nanomicelles exhibited good tissue stickiness that can release curcumin for a long time. Moreover, full-thickness excision models and linear incision models were applied to analyze wounds' in vivo healing. Histopathologic examination showed that curcumin load nanomicelles could enhance cutaneous wound repair. It is concluded that biodegradable curcumin encapsulated nanomicelles have significant potential for wound healing [85].

$\mathrm{Xu}$ et al., paclitaxel-loaded micelles were developed, and hydrogel was formulated while loading these nanoparticles for skin melanoma treatment. Skin penetration and retention study were performed for the following formulation. The cell lines studied showed more cellular uptake in B16 melanoma cell lines, and in vivo studies in B16 cells showed preferable anticancer activity than free taxol [86].

Rosa Juliana et al. develop a new approach for gene therapy for various skin diseases. The RNA interferences used for gene therapy has been considered as the most novel techniques for individualized therapy. In several skin diseases, siRNA application was established against skin cancer, psoriasis, dermatitis, and leprosy. The following approach used siRNA encapsulated nanomicelles for topical delivery. It acted via inhibition of expression of the transcript that has potential for gene suppression [87].

Bingxin et al. developed oridonin (ORI) encapsulated nanomicelles by utilizing monomethoxy poly(ethylene glycol)-poly( $\varepsilon$-caprolactone). The study revealed that ORI was encapsulated into the core-shell structure of micelles that retained its anti-cancer activity. The ORI could be released from the nanomicelles in a sustained way for in vitro study. The results concluded that ORI encapsulated micelles showed potential for intravenous and transdermal drug delivery in cancer therapy [88]. Anup Jose et al. developed cationic liposomes encapsulated with curcumin and complexed with STAT3 siRNA. The results concluded that the liposome-siRNA encapsulated with curcumin was taken up by clathrin modulated endocytosis.

The co-encapsulation of STAT3 siRNA along with curcumin have a higher potential for cancer cell apoptosis and inhibition with STAT3 siRNA and free curcumin. It is concluded that cationic liposomes are non-invasive iontophoretic delivery of both co-encapsulated substances to target the skin malignant cells [89].

\subsection{Gold nanoparticles.}

Gold nanoparticles (AuNPs) are the most used and applied platforms for treating, diagnosing, and monitoring skin disease, including skin tumors [90]. Limon et al. developed gold nanoparticles (GNP) coated with thiolates that highly solubilize in water. The uptake and internalization of fluorophore coated AuNPs in keratinocytes of human was elaborated using confocal fluorescence microscopy.

The functionalized nanoparticles inhibited intracellular KLK5 and HaCaT activity and diminished the IL- 8 secretion stimulated by TLR-2 ligands. The following study showed that GNPs have a significant ability for new intracellular delivery of drugs and antibodies in treating skin cancer other diseases, including Rosacea [91]. Preet et al. demonstrated the development of nisin gold nanoparticles loaded with doxorubicin for murine skin cancer treatment. The results indicate a substantial reduction in tumor volume when calculated at the termination of 
therapy. Therefore, the potential decline in the serum values of all evaluated cytokines NF- $\kappa \beta$, TNF- $\alpha$, TNF- $\beta$, IL-10. And a potential increase in tissue ROS (reactive oxygen species) levels and lipid peroxides, and superoxide dismutase in various treatment groups. The following study showed that the AuNPs assisted co-delivery of nisin doxorubicin for murine cancer cells and DMBA cells in cultured cells in the lab [92].

Nirmala et al. developed the AuNPs considered for apoptosis and anti-malignant efficacy at the minimum inhibitory concentration. The cytotoxicity of the gold nanoparticles can synergize the effects of the phenolic compound of the V.vinifera and the efficacy of the conjugated AuNPs that causes necrosis and tumor cell death in following cell lines studies [93].

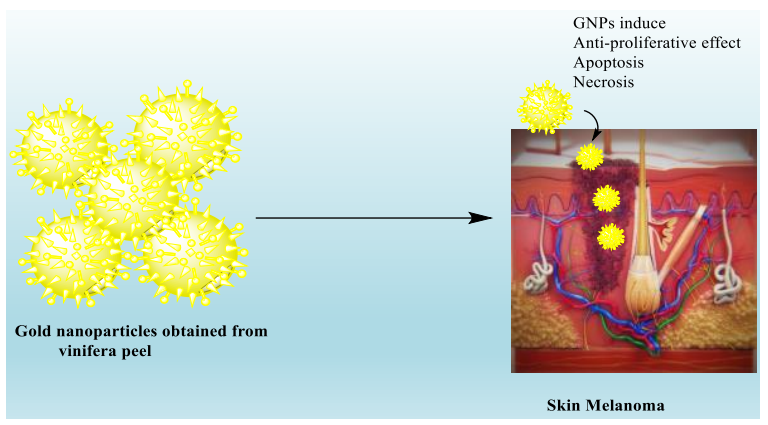

Figure 3. Gold nanoparticles obtained from vinifera peel induce antiproliferative, apoptosis, and necrosis in melanoma cell lines.

Fratoddi et al. developed the functionalized gold nanoparticles encapsulated with MTX (methotrexate) to treat moderate to severe inflammatory diseases. The efficacy of the AuNPs of the gold nanoparticles was loaded with MTX topically administered on the skin and checked on skin models in vivo and in vitro in the psoriasis mice model. The cell proliferation rate, inflammation, and epidermal thickness were also evaluated - the K6CD3, Ki67, and CD8 used for immunochemistry evaluation showed reduced staining in AuNPs. Blood analysis showed no distinctive differences in blood cell count level and ALT, AST levels at the start, and treatment termination. Topical gold nanoparticles therapy can stimulate the decrease in epidermal thickness, reduction in keratinocyte multiplications, and inflammatory infiltration in the psoriasis mice model [94].

\subsection{Mesoporous silica nanoparticles.}

Mesoporous silica nanoparticles (MeSNPs) are a wide range of functionalities and are modular as they can be modified to fit almost any setting. These nanoparticles have a greater surface to volume ratio that increased the functionalization while maintaining the porosity that allows the inorganic platform to an appreciable amount of carrier without destabilization of silica framework. The recent advancement in nano-medicines helps to develop mesoporous silica nanoparticles at a large scale. There are several studies that showed the use of MeSNPs in skin carcinoma [95].

Hirai et al. evaluated the efficiency of nSPs (amorphous silica nanoparticles), one of the broadly used nanomaterials that can induce the targeted effect by penetrating the skin. The mice having atopic dermatitis (AD) (via dermal injection antigen Dermatophagoides pteronyssinus) [96]. It is evaluated that amorphous silica nanoparticles can produce immunemodulating effects that can penetrate the skin. However, nSPs could produce a risk for augmentation of immune diseases related to skin. Histopathological results showed that Dp and 
silica particles produce augmentation of AD. Moreover, the results are related to skin infections induce by thymic lymphopoietin and IL-18 [96].

Lio et al., interfering RNA (siRNA) encapsulated mesoporous silica nanoparticles applied to treat skin diseases that include SCC (squamous cell carcinoma). To study the effects of silica nanoparticles topically to the SCC in a mouse model. MeSNPs encapsulated with TGFßR-1 siRNA show a two-fold suppression of TGFßR-1 [97]. Xing Ma et al. developed 5aminolevulinic acid (5-ALA) encapsulated MeSNPs were produced to transport 5-ALA for (PDT) photodynamic therapy against skin cancer cell lines B16F10. The folic acid attached to the surface of 5-ALA encapsulated HMeSNPs enabled the endocytosis of NPs in cancer cell lines. Figure 4 describe the targeting of skin cancer cell via mesoporous nanoparticles [98].

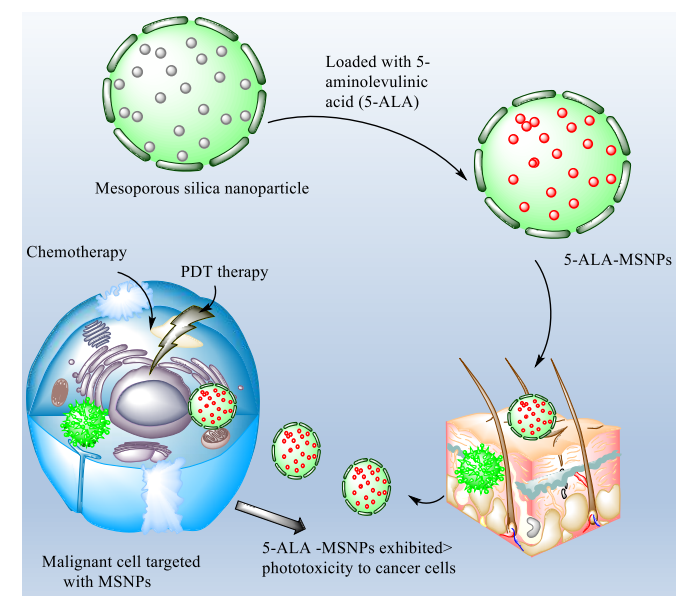

Figure 4. 5-aminolevulinic acid (5-ALA) encapsulated mesoporous silica nanoparticles for Photodynamic therapy against skin cancer cells (B16F10).

P.Scodeller et al. developed silica nanoparticles (SiNPs) via self-assembly layer methodology. The NPs were analyzed as adjuvant of carboplatin, intervene in A375 cell lines of human skin cancer having mice and compared with another enzyme (inactivated). It is stated that the volume of the tumor with SiNPs inactivated hyal was potentially increased in comparison to the inactivated one. The matrix distorting enzymes are inactivated on SiNPs and are a more efficient cancer drug aid than the inactivated enzyme [99].

Rosenholm et al. developed the MeSNPs and highlighted the new drug delivery way. In vivo and in vitro, the therapeutic benefit of these systems for in vivo use has been studied. Moreover, the results showed that the cellular studies could be replicated along with targeting and safety issues in cancer therapy [100]. Fiorenza Rancan et al. evaluated the skin permeation and uptake of SiNPs. The topical application of nanoparticles was linked with dendritic and epidermis cells independent of their functionalization. The results concluded that, in patients with eczema and atopic dermatitis, skin layers' analysis is highly significant to assess the penetration of nanoparticles [101].

\subsection{Iron oxide nanoparticles.}

The superparamagnetic iron-oxide NPs (SPION) for drug targeting and other biomedical implementation have been evaluated because of greater drug encapsulating capacity, intense magnetic responsiveness, and greater targeted delivery efficiency. Literature suggested that skin composition and structure will not approve the materials greater than 600 $\mathrm{Da}(>600 \mathrm{Da})$ to pass through the skin layers. The recent advances in nanomedicines have made it easy to develop different types of MNPs (magnetic NPs) with few nanometers. Many studies 
demonstrated that these types of special nanomaterials help to permeate the stratum corneum and hair follicles via the epidermis [102].

Rao et al. developed the EPI-SPION (epirubicin encapsulated iron oxide NPs) to target skin carcinoma via transdermal route. The modifications of the SPION results in a drug carrier that applied magnetism for chemotherapy of skin cancer. The cell lines study of skin cancer in WM266 and HaCaT keratinocyte cells showed that SPION has good compatibility. The in vitro study evaluated that the EPI loaded SPION can permeate the skin. The study concluded that the potential of iron oxide NPs for easy transdermal delivery in skin melanoma [103].

Cengelli et al. studied the interactions of biocompatible cationic amino ultra-small superparamagnetic iron oxide nanoparticles (USPIONs) with human cells in different dimension cultures (2D and 3D) using electron microscopy and biochemical techniques. The results showed internalization of the amino-SPIONs by human melanoma cells. The uptake pathway was clathrin modulated and localized in the lysosome, which induces the stimulation and decreases the expression of the cathepsin D and transferrin receptors in skin fibroblasts under evaluation of skin melanoma [104].

Choi et al. studied the photon-activated therapy for skin melanoma and the potential of the sensitizing effect of iron oxide NPs. X-rays photon stimulated therapy was evaluated by targeting the CT26 tumor-bearing mice. The decrease in viability and damage was seen in the FeO-NPs treated cell lines. The results concluded that iron oxide nanoparticles would enhance the therapeutic effect with a low concentration that may have potential and significant treatment for the skin's malignancies [105].

Musazzi et al. developed the semi-solid formulation with iron oxide NPs for human skin penetration to target different skin diseases. The in vitro penetration of IONs aggregates was evaluated by utilizing isolated stratum corneum (SC) or humans' epidermis. The semisolid preparation of different FeO-NPs permeation as compared to the aqueous suspension. The Cet cream formulation enhances the penetration, and low retention amount compared to the cold cream and favored the accumulation into the skin membrane. The results concluded that the skin semi-solid formulation could be used to deliver the FeO-NPs without disturbing the permeation pattern on the skin surface [106].

\section{Results and Discussion}

When normal cells transform and spread out of control, cancer starts and creates a tumor. Skin cancer is diagnosed for 3 million Americans each year, making this cancer one of the most common cancer kinds in this country and also in the world. If skin cancer is detected early, topical treatments, procedures performed in a dermatologist's office, or ambulatory surgery may typically be used to treat it [107]. Skin cancer usually kills 1 percent of all cancer types in the world. Skin cancer can be more severe in some situations and needs treatment by a multi-modal approach that needs dermatologists and oncologists experts together [108]. Skin cancer usually showed 4 types of cancer that include squamous, basal, Merkel, and melanoma cell carcinoma [109]. Melanoma began with melanocytes and was reported as the most deadly skin cancer. There are some drawbacks to conventional methods. The advancement of new strategies for the detection of skin cancer is vital [110]. The nanosensor is an analytical instrument used for the identification of a biological substance. Nanomedicine is playing an increasingly significant role in the production of nanosensors. The biosensor or nano biosensor science is widely used to detect biomarkers in skin cancer [111]. It can be claimed that with the emergence of Nanomedicine and its effect on the production of ultrasensitive instruments, 
it is possibly one of the most promising solutions to fix some of the problems related to the growing need to create an extremely sensitive, quick, and economical method of a skin cancer diagnosis. Different electrochemical and optical biosensors have been developed for skin cancer detection that will be discussed below.

\subsection{Biomarkers in skin cancer.}

The identification of biomarkers associated with skin cancer has provided new insights into the area of theranostics cancer. One of the earliest prognostic biomarkers of melanoma is lactate dehydrogenase (LDH) [112]. Another prognostic biomarker in metastatic skin cancer patients is serum S100B levels [113]. The BRAF V600 can be the best biomarker for metastatic melanoma. Recognition of tyrosinase (TYR) mRNAs in serum or blood is another biomarker for metastatic melanoma [114]. In inflammatory problems, over-expression of cyclooxygenase- 2 is a promising biomarker for tumor inflammogenesis, metastasis, and angiogenesis [115]. Microsomal prostaglandin E2 synthase-1 was reported in some studies as a predictor of melanoma or skin cancer [116]. A key role in melanoma progression has been documented to be played by matrix metalloproteinases ( MMPs) [117].

Many cancers, including malignant melanoma, calcium-binding proteins of S100 group such as S100B, S100A9, and S100A4, have attracted considerable interest as novel biomarkers [118]. It is often undetectable in the early stages of melanoma. In contrast, late-stage melanoma displays substantial circulating tumor DNA (ctDNA) levels [119]. The potential biomarkers of melanoma and other pathologies can be microRNAs or long noncoding RNAs [120]. The exosomes of patients with metastatic melanoma were extracted from miRNA19a, miRNA21, miRNA149, miRNA17, and miRNA126 [121].

Collectively, the discovery of biomarkers derived from liquid biopsies is not as fast as in comparison to genomics and genomics biomarkers. Also, many proposed biomarkers in the clinical application may not have adequate validity. Among the factors that limit the use of ideal biomarkers in POC, applications are the absence of repeatability, optimization procedures, and process duration. Previous to marketing, biomarkers must meet strict laws by regulatory authorities.

\subsection{Traditional approaches to the diagnosis of skin cancer.}

Dermoscopy, self-skin examination, optical coherence tomography, and ultrasonography are traditional skin cancer detection [122]. A Skin Self-Exam (SSE) is a skin examination that you do on your own. It is a method to detect any alterations in the skin or irregular areas. SSE is a simple method of detecting skin cancer with a 6-50\% prevalence of self-diagnosed skin cancer incidence [123]. In dermoscopy, optical magnification (*10) and fluid immersion were used to view skin lesions as a non-invasive imaging process. From mild to malignant melanoma, dermoscopy illustrates the development of skin lesions. Compared to a naked eye examination, dermoscopy improves the susceptibility and precision of detecting melanoma to around 20 and $10 \%$, respectively [124].

Another traditional technique that microscopically evaluates melanoma at the papillary dermis level and epidermis is reflectance confocal microscopy or RCM [125]. Different reflectance indexes of skin cells and their anatomical structure generate contrasts of the final image in the resulted photograph. For melanin and keratin, the maximum contrast is with a reflection index of 1.7 and 1.5, respectively. High-frequency ultrasonography has created high- 
resolution images to distinguish different forms of skin cancer. This high resolution (frequencies up to $100 \mathrm{MHz}$ ) helps the system to picture the cutaneous components, epidermis and dermis, and vessels to the diagnosis of skin cancer [124]. In ultrasound tests, the appearance of a healthy, hypoechogenic, and thick tissue suggests the existence of skin cancer. In diagnosing skin cancer, infrared light was applied in optical coherence tomography to create a no invasive process. In cases of misunderstanding with ultrasonography, OCT is appropriate. OCT, though, only investigates tumor lesions below $1 \mathrm{~mm}$ [126].

In the diagnosis of skin cancer, these conventional techniques have been developed, but significant disadvantages have restricted their clinical application. Many of these approaches are time-consuming, costly, and need an experienced expert.

\subsection{Electrochemical nanosensors.}

Biosensors are commonly used instruments for electrochemical biomarker detection and can theoretically be used to establish cancer diagnostic with point-of-care checks [127]. An efficient and low-cost detection technique for melanoma cancer diagnosis is the electrochemical identification of functional melanoma biomarkers, which significantly optimizes sensitivity and specificity [128]. Immunosensors are effective for cancer detection and are produced using electrochemical transducer-anchored antibodies. A significant part of the creation of electrochemical immunosensors is expected to be the manufacturing of responsive and stable materials by immobilizing biomolecules. The use of nanoparticles in an immunosensor also enables biological macromolecules with high biological stability and activity to be effectively immobilized due to their high adsorption ability and wide surface area [129, 130]. Seenivasan et al. developed a new immunosensor based on $\mathrm{SiO}_{2} \mathrm{NPs}$ and polypyrrole nanocomposite for early detection of melanoma cancer [131].

The particular strategy was based on the attachment between the anti-MC1R antibody and the melanocortin 1 receptor antigen on the cell's surface. The suggested immunosensor was particularly sensitive with a sensitivity of 20 cells $/ \mathrm{mL}$, accurate and repeatable, and the readyto-use electrode loaded with Ab was lasted for 14 days. In another study, Xiang et al. mentioned a combined nano-mesoporous $\mathrm{Co}_{3} \mathrm{O}_{4}$ sheet $\left(\mathrm{Au} / \mathrm{Co}_{3} \mathrm{O}_{4}\right)$ and aminated graphene (GS-NH2) immunosensor based on $\mathrm{Au}$ NPs for melanoma adhesion molecule antigen (CD146) identification [132]. With a LOD of $3.4 \mathrm{pg} / \mathrm{mL}$ and $\mathrm{S} / \mathrm{N}$ of 3, the nano sensor-operated over a line range of 0.01-15 $\mathrm{ng} / \mathrm{mL}$.

Wearable sensors have gained tremendous interest in recent years. Different wearable electrochemical or optical sensors have been developed to monitor body fluids such as saliva or sweat in real-time [133].

For the rapid screening of skin melanoma, Bianca et al. developed a portable bendable nanosensor and a non-invasive micro-needle sensor [134]. The wearable electrochemical sensors were able to identify the existence of tyrosinase (TYR as skin cancer biomarker). The elastic sensor showed strong mechanical deformation resistance, whereas the hollow microneedle unit was filled with carbon paste coated with catechol to measure the low levels of TYR in tissue. Pretty biased measurement was obtained by adsorbing the TYR enzyme marker's CAT substrate and amperometrically measuring the BQ reaction product at a low-potential of $-0.25 \mathrm{~V}$. The resultant epidermal bandage and wearable microneedle sensors showed good analytical efficiency and provided significant promise to monitor the TYR biomarker on both the skin surface and inside the skin. 
Electric cell-substrate impedance sensing (ECIS) and Electrochemical impedance spectroscopy (EIS) are the common methods currently available for characterizing single cells based on a specific frequency range of cellular electrical answers and long-term cell physiological answers [135]. The EIS system has been commonly reported to detect skin cancer. It includes a disposable electrode probe that differentiates between abnormal and normal skin lesions using electrical impendence variations [136]. In this regard, A highly sensitive electrochemical impedance spectroscopy (EIS) approach has been documented by Prathap et al., useful in identifying melanoma cells precisely and quickly using anti-MC1R antibody attached PANI-NFs [137]. A perfect linear correlation was obtained between the current and the target cell (density of 15 to 7000 cells $/ 5 \mathrm{~mL}$ and LOD of 1 cell $/ \mathrm{mL}$ ).

Silicon nanowire functionalized with antibody field-effect transistors demonstrated high specificity to analyte identification, allowing analyte concentrations to be sensed at levels not easily available by other tools [138]. Identifying serum melanoma biomarkers, such as the newly found tumor necrosis factor receptor superfamily participant TROY (TNFRSF19), is at low amounts, is an important factor. The identification of TROY in the buffer with various concentrations and silicon nanowires was reported by Maedler et al. The sensitivity of nanosensors was evaluated by comparing the signal with that acquired from BSA in the buffer solution [139]. To differentiate the two signals, both the signal size and the reaction kinetics were applied. They obtained the dissociation and interaction constants for the reaction of TROY and antibody by their reaction models.

Despite the benefits of using electrochemical approaches, skin cancer identification at its early phases needs to study human body fluid with deficient concentrations. To enhance the therapeutic potential and diagnostic precision, the combination of multimodal imaging and therapeutic features within a nanoplatform is essential. Integrating electrochemical approaches with recently designed electro-active complexes and electrochemical sensing nanoparticles can further increase the limits of detection and susceptibility for new biomarkers. Even more developments in nanoscience, bioengineering, molecular biology, and computational biology are expected to open the path to producing accurate electrochemical biosensors to enhance customized treatment strategies for some diseases.

\subsection{Optical nanosensors.}

As novel disease biosensors, optical techniques have received considerable attention in recent years [140]. As a symptom of dermatological diseases, including melanoma, increased tyrosinase activation is a marker. In melanin biosynthesis, tyrosinase (TYR) is an important biomarker for detecting actinic injury, vitiligo, and melanoma [141]. Hu et al. published a simple fluorescent experiment-based carbon quantum dots attached with dopamine (CQDsDopa) for TYR activation [142]. Two wide linear regions were identified by the experiment (711.1-2925.4 U/L and 44.4-711.1 U/L) and LOD of 17.7 U/L. In blood serum, the suggested fluorescent analysis was used to test TYR activity.

In other studies, Li et al. developed an oligonucleotide fluorescent probe with pyrene alteration and in-situ integration of nucleoside phosphoramidites (T-C-A-G) with phosphorus modification of pyrene at the internal phosphate backbone [143]. They demonstrated that the inclusion of exactly aligned analytes caused the fluorescence intensity to increase quickly (less than $20 \mathrm{~s}$ ) and significantly (up to 23.5 times), whereas analytes with a single mismatch on both sides of pyrene attached phosphate showed a steady fluorescence intensity level similar to that of the background. As a practical optical imaging tool, fluorescent-labeled antibodies 
have been applied in the diagnosis of microscopic melanoma. In another study, Larissa et al. showed that antiangiogenic therapy's addition increases the absorption of monoclonal antibodies labeled fluorescently within melanoma tumors [144].

One of the favorite new investigations that have drawn great interest from researchers because of its economical price and good sensitivity is the photoelectrochemical (PEC) biosensing technique [145]. In this light, a high sensitive photoelectrochemical nanosensor was reported by Erhu et al. for DNA detection. The nanosensor was based on cascade signal amplification (hybridization chain reaction, catalytic hairpin assembly, and alkaline phosphatase) [146]. The T-DNA was detected with LOD of $0.052 \mathrm{fM}$ and linear response range of $0.1 \mathrm{fM}-100 \mathrm{pM}$. The suggested PEC biosensor demonstrated excellent analytical efficiency. In another analysis, Lopes et al. prepared biocompatible Au NPs covered with oleic acid and hyaluronic acid and used in a melanoma cell line (B16F10), keratinocytes, and also Saccharomyces cerevisiae [147]. Au NPs showed a near-infrared absorption in 750-1400 nm. The covered Au NPs also demonstrated a spherical shape with an average diameter of $297 \mathrm{~nm}$ without toxic effect on the yeast and cell lines tested. Nonetheless, the viability of the B16F10 cell line was decreased down to $20 \%$ after laser application. This study showed promising results for melanoma treatment and diagnosis.

\section{Conclusions and Future Prospectives}

Nano dermatology is a novel area of research having applications in the field of medicines. Many nanomaterials have been implemented in modern society. Updated versions are developed at a phenomenal rate as some inorganic nanomaterials coupled to label as antibodies and have proven efficacy for visualizing various skin diseases and skin tumors.

Skin cancer is fast-growing cancer that some vital consideration is required for quick and precise diagnostic tools. However, despite the outstanding role of conventional methods in reducing melanoma's mortality rate, the limitations of these methods for clinical use and recent rapid advancement in optical and electrochemical nanosensors have led to innovative approaches for detecting melanoma in trial applications.

Effective clinical implementation of the methods of detection demands that they be incorporated with POC instruments. The important obstacle for electrochemical melanoma detection is that no biomarker has been showing a perfect signal. Some bio-markers appear to have low selectivity, raising the likelihood of erroneous results.

Assimilating electrochemical methods with recently created electro-active compounds and electrochemical biosensors would expand the limits of detection and sensitivity for newer biomarkers. Furthermore, nanomaterials tend to have a synergistic effect between bioactivity, permeability, and catalytic potential to improve the final signal. Further advances in medical technology, genetic engineering, computational biology, and molecular biology are expected to open the door to the production of effective electrochemical biosensors to enhance customized treatment strategies for some diseases.

\section{Funding}

This research received no external funding.

\section{Acknowledgments}

This research has no acknowledgment. 


\section{Conflicts of Interest}

\section{The authors declare no conflict of interest.}

\section{References}

1. Esteva, A.; Kuprel, B.; Novoa, R. A.; Ko, J.; Swetter, S.M.; Blau, H.M.; Thrun, S. Dermatologist-level classification of skin cancer with deep neural networks. Nature 2017, 542, 115-118, https://doi.org/10.1038/nature21056.

2. Cullen, J.K.; Simmons, J.L.; Parsons, P.G.; Boyle, G.M. Topical treatments for skin cancer. Advanced drug delivery reviews 2020, 153, 54-64, https://doi.org/10.1016/j.addr.2019.11.002.

3. Rogers, H.W.; Weinstock, M.A.; Feldman, S.R.; Coldiron, B.M. Incidence estimate of nonmelanoma skin cancer (keratinocyte carcinomas) in the US population, 2012. JAMA dermatology 2015, 151, 1081-1086, https://doi.org/10.1001/jamadermatol.2015.1187.

4. Bray, F.; Ferlay, J.; Soerjomataram, I.; Siegel, R.L.; Torre, L.A.; Jemal, A. Global cancer statistics 2018: GLOBOCAN estimates of incidence and mortality worldwide for 36 cancers in 185 countries. CA: a cancer journal for clinicians 2018, 68, 394-424, https://doi.org/10.3322/caac.21492.

5. Padya, B.S.; Pandey, A.; Pisay, M.; Koteshwara, K.B.; Chandrashekhar Hariharapura, R.; Bhat, K.U.; Biswas, S.; Mutalik, S. Stimuli-responsive and cellular targeted nanoplatforms for multimodal therapy of skin cancer. European Journal of Pharmacology 2021, 890, https://doi.org/10.1016/j.ejphar.2020.173633.

6. Wollina, U.; Schreiber, A.; Merla, K.; Haroske, G. Combined cetuximab and volumetric modulated arcradiotherapy in advanced recurrent squamous cell carcinoma of the scalp. Dermatology Reports 2011, 3, https://doi.org/10.4081/dr.2011.e57.

7. Smith, V.; Walton, S. Treatment of facial basal cell carcinoma: a review. Journal of skin cancer 2011, 2011, https://doi.org/10.1155/2011/380371.

8. Bilal, M.; Iqbal, H. New Insights on Unique Features and Role of Nanostructured Materials in Cosmetics. Cosmetics 2020, 7, https://doi.org/10.3390/cosmetics7020024.

9. Rahdar, A.; Hajinezhad, M.R.; Bilal, M.; Askari, F.; Kyzas, G.Z. Behavioral effects of zinc oxide nanoparticles on the brain of rats. Inorganic Chemistry Communications 2020, 119, https://doi.org/10.1016/j.inoche.2020.108131.

10. Raza, A.; Hayat, U.; Bilal, M.; Iqbal, H.M.; Wang, J.-Y. Zein-based micro-and nano-constructs and biologically therapeutic cues with multi-functionalities for oral drug delivery systems. Journal of Drug Delivery Science and Technology 2020, 58, https://doi.org/10.1016/j.jddst.2020.101818.

11. Sparreboom, A.; Scripture, C.D.; Trieu, V.; Williams, P.J.; De, T.; Yang, A.; Beals, B.; Figg, W.D.; Hawkins, M.; Desai, N. Comparative preclinical and clinical pharmacokinetics of a cremophor-free, nanoparticle albumin-bound paclitaxel (ABI-007) and paclitaxel formulated in Cremophor (Taxol). Clinical cancer research 2005, 11, 4136-4143, https://doi.org/10.1158/1078-0432.CCR-04-2291.

12. Wang, R.; Billone, P.S.; Mullett, W.M. Nanomedicine in action: An overview of cancer nanomedicine on the market and in clinical trials. Journal of Nanomaterials 2013, 2013, https://doi.org/10.1155/2013/629681.

13. Zhao, M.; Li, H.; Ma, Y.; Gong, H.; Yang, S.; Fang, Q.; Hu, Z. Nanoparticle abraxane possesses impaired proliferation in A549 cells due to the underexpression of glucosamine 6-phosphate N-acetyltransferase 1 (GNPNAT1/GNA1). International Journal of Nanomedicine 2017, 12, https://doi.org/10.2147/IJN.S129976.

14. Nguyen, T.X.; Huang, L.; Gauthier, M.; Yang, G.; Wang, Q. Recent advances in liposome surface modification for oral drug delivery. Nanomedicine 2016, 11, 1169-1185, https://doi.org/10.2217/nnm.16.9.

15. Anselmo, A.C.; Mitragotri, S. Nanoparticles in the clinic. Bioengineering \& translational medicine 2016, 1 , 10-29, https://doi.org/10.1002/btm2.10003.

16. Zhao, Z.; Ukidve, A.; Krishnan, V.; Mitragotri, S. Effect of physicochemical and surface properties on in vivo fate of drug nanocarriers. Advanced Drug Delivery Reviews 2019, 143, 3-21, https://doi.org/10.1016/j.addr.2019.01.002.

17. Rasheed, T.; Nabeel, F.; Raza, A.; Bilal, M.; Iqbal, H.M.N. Biomimetic nanostructures/cues as drug delivery systems: a review. Materials Today Chemistry 2019, 13, 147-157, https://doi.org/10.1016/j.mtchem.2019.06.001.

18. Munir, S.; Shah, A.A.; Rahman, H.; Bilal, M.; Rajoka, M.S.R.; Khan, A.A.; Khurshid, M. Nanozymes for medical biotechnology and its potential applications in biosensing and nanotherapeutics. Biotechnology Letters 2020, 42, 357-373, https://doi.org/10.1007/s10529-020-02795-3.

19. Ain, Q.-U.; Munir, H.; Jelani, F.; Anjum, F.; Bilal, M. Antibacterial potential of biomaterial derived nanoparticles for drug delivery application. Materials Research Express 2020, 6, https://doi.org/10.1088/2053-1591/ab715d.

20. Bilal, M.; Barani, M.; Sabir, F.; Rahdar, A.; Kyzas, G.Z. Nanomaterials for the treatment and diagnosis of Alzheimer's disease: An overview. NanoImpact 2020, 20, https://doi.org/10.1016/j.impact.2020.100251.

21. Barani, M.; Mirzaei, M.; Torkzadeh-Mahani, M.; Adeli-sardou, M. Evaluation of Carum-loaded Niosomes on Breast Cancer Cells:Physicochemical Properties, In Vitro Cytotoxicity, Flow Cytometric, DNA 
Fragmentation and Cell Migration Assay. Scientific Reports 2019, 9, 1-10, https://doi.org/10.1038/s41598019-43755-w.

22. Barani, M.; Mirzaei, M.; Torkzadeh-Mahani, M.; Lohrasbi-Nejad, A.; Nematollahi, M.H. A new formulation of hydrophobin-coated niosome as a drug carrier to cancer cells. Materials Science and Engineering: C 2020, 113, https://doi.org/10.1016/j.msec.2020.110975.

23. Barani, M.; Mirzaei, M.; Torkzadeh-Mahani, M.; Nematollahi, M.H. Lawsone-loaded Niosome and its antitumor activity in MCF-7 breast Cancer cell line: a Nano-herbal treatment for Cancer. Daru : journal of Faculty of Pharmacy, Tehran University of Medical Sciences 2018, 26, 11-17, https://doi.orrg/10.1007/s40199-018-0207-3.

24. Barani, M.; Nematollahi, M.H.; Zaboli, M.; Mirzaei, M.; Torkzadeh-Mahani, M.; Pardakhty, A.; Karam, G.A. In silico and in vitro study of magnetic niosomes for gene delivery: The effect of ergosterol and cholesterol. Materials Science and Engineering: C 2019, 94, 234-246, https://doi.org/10.1016/j.msec.2018.09.026.

25. Barani, M.; Sabir, F.; Rahdar, A.; Arshad, R.; Kyzas, G.Z. Nanotreatment and Nanodiagnosis of Prostate Cancer: Recent Updates. Nanomaterials 2020, 10, https://doi.org/10.3390/nano10091696.

26. Barani, M.; Torkzadeh-Mahani, M.; Mirzaei, M.; Nematollahi, M.H. Comprehensive Evaluation of Gene Expression in Negative and Positive Trigger-based Targeting Niosomes in HEK-293 Cell Line. Iranian Journal of Pharmaceutical Research 2020, 19, 166-180, https://doi.org/10.22037/ijpr.2019.112058.13507.

27. Das, S.S.; Bharadwaj, P.; Bilal, M.; Barani, M.; Rahdar, A.; Taboada, P.; Bungau, S.; Kyzas, G.Z. StimuliResponsive Polymeric Nanocarriers for Drug Delivery, Imaging, and Theragnosis. Polymers 2020, 12, https://doi.org/10.3390/polym12061397.

28. Davarpanah, F.; Khalili Yazdi, A.; Barani, M.; Mirzaei, M.; Torkzadeh-Mahani, M. Magnetic delivery of antitumor carboplatin by using PEGylated-Niosomes. Daru : journal of Faculty of Pharmacy, Tehran University of Medical Sciences 2018, 26, 57-64, https://doi.org/10.1007/s40199-018-0215-3.

29. Ebrahimi, A.K.; Barani, M.; Sheikhshoaie, I. Fabrication of a new superparamagnetic metal-organic framework with core-shell nanocomposite structures: Characterization, biocompatibility, and drug release study. Materials Science and Engineering: $\quad$ C 2018, 92, 349-355, https://doi.org/10.1016/j.msec.2018.07.010.

30. Hajizadeh, M.R.; Maleki, H.; Barani, M.; Fahmidehkar, M.A.; Mahmoodi, M.; Torkzadeh-Mahani, M. In vitro cytotoxicity assay of D-limonene niosomes: an efficient nano-carrier for enhancing solubility of plantextracted agents. Research in pharmaceutical sciences 2019, 14, 448-458, https://doi.org/10.4103/17355362.268206.

31. Hajizadeh, M.R.; Parvaz, N.; Barani, M.; Khoshdel, A.; Fahmidehkar, M.A.; Mahmoodi, M.; TorkzadehMahani, M. Diosgenin-loaded niosome as an effective phytochemical nanocarrier: physicochemical characterization, loading efficiency, and cytotoxicity assay. DARU Journal of Pharmaceutical Sciences 2019, 27, 329-339, https://doi.org/10.1007/s40199-019-00277-0.

32. Rahdar, A.; Hajinezhad, M.R.; Nasri, S.; Beyzaei, H.; Barani, M.; Trant, J.F. The synthesis of methotrexateloaded F127 microemulsions and their in vivo toxicity in a rat model. Journal of Molecular Liquids 2020, 313, https://doi.org/10.1016/j.molliq.2020.113449.

33. Rahdar, A.; Taboada, P.; Hajinezhad, M.R.; Barani, M.; Beyzaei, H. Effect of tocopherol on the properties of Pluronic F127 microemulsions: Physico-chemical characterization and in vivo toxicity. Journal of Molecular Liquids 2019, 277, 624-630, https://doi.org/10.1016/j.molliq.2018.12.074.

34. Torkzadeh-Mahani, M.; Zaboli, M.; Barani, M.; Torkzadeh-Mahani, M. A combined theoretical and experimental study to improve the thermal stability of recombinant D-lactate dehydrogenase immobilized on a novel superparamagnetic Fe3O4NPs@metal-organic framework. Applied Organometallic Chemistry 2020, 34, https://doi.org/10.1002/aoc.5581.

35. Rahdar, A.; Aliahmad, M.; Samani, M.; HeidariMajd, M.; Susan, M.A.B.H. Synthesis and characterization of highly efficacious Fe-doped ceria nanoparticles for cytotoxic and antifungal activity. Ceramics International 2019, 45, 7950-7955, https://doi.org/10.1016/j.ceramint.2019.01.108.

36. Rahdar, A.; Hajinezhad, M.R.; Sivasankarapillai, V.S.; Askari, F.; Noura, M.; Kyzas, G.Z. Synthesis, characterization, and intraperitoneal biochemical studies of zinc oxide nanoparticles in Rattus norvegicus. Applied Physics A 2020, 126, 1-9, https://doi.org/10.1007/s00339-020-03535-0.

37. Taimoory, S.M.; Rahdar, A.; Aliahmad, M.; Sadeghfar, F.; Hajinezhad, M.R.; Jahantigh, M.; Shahbazi, P.; Trant, J.F. The synthesis and characterization of a magnetite nanoparticle with potent antibacterial activity and low mammalian toxicity. Journal of Molecular Liquids 2018, 265, 96-104, https://doi.org/10.1016/j.molliq.2018.05.105.

38. Nikazar, S.; Sivasankarapillai, V.S.; Rahdar, A.; Gasmi, S.; Anumol, P.S.; Shanavas, M.S. Revisiting the cytotoxicity of quantum dots: an in-depth overview. Biophysical Reviews 2020, 12, 703-718, https://doi.org/10.1007/s12551-020-00653-0.

39. Pillai, A.M.; Sivasankarapillai, V.S.; Rahdar, A.; Joseph, J.; Sadeghfar, F.; Anuf A, R.; Rajesh, K.; Kyzas, G.Z. Green synthesis and characterization of zinc oxide nanoparticles with antibacterial and antifungal activity. Journal of Molecular Structure 2020, 1211, https://doi.org/10.1016/j.molstruc.2020.128107. 
40. Rahdar, A.; Hajinezhad, M.R.; Hamishekar, H.; Ghamkhari, A.; Kyzas, G.Z. Copolymer/graphene oxide nanocomposites as potential anticancer agents. Polymer Bulletin 2020, 1-22, https://doi.org/10.1007/s00289020-03354-6.

41. Saravani, R.; Sargazi, S.; Saravani, R.; Rabbani, M.; Rahdar, A.; Taboada, P. Newly crocin-coated magnetite nanoparticles induce apoptosis and decrease VEGF expression in breast carcinoma cells. Journal of Drug Delivery Science and Technology 2020, 60, https://doi.org/10.1016/j.jddst.2020.101987.

42. Sivasankarapillai, V.S.; Somakumar, A.K.; Joseph, J.; Nikazar, S.; Rahdar, A.; Kyzas, G.Z. Cancer theranostic applications of MXene nanomaterials: Recent updates. Nano-Structures \& Nano-Objects 2020, 22, https://doi.org/10.1016/j.nanoso.2020.100457.

43. Sivasankarapillai, V.S.; Pillai, A.M.; Rahdar, A.; Sobha, A.P.; Das, S.S.; Mitropoulos, A.C.; Mokarrar, M.H.; Kyzas, G.Z. On Facing the SARS-CoV-2 (COVID-19) with Combination of Nanomaterials and Medicine: Possible Strategies and First Challenges. Nanomaterials 2020, 10, https://doi.org/10.3390/nano10050852.

44. Sayadi, K.; Rahdar, A.; Hajinezhad, M.R.; Nikazar, S.; Susan, M.A.B.H. Atorvastatin-loaded SBA-16 nanostructures: Synthesis, physical characterization, and biochemical alterations in hyperlipidemic rats. Journal of Molecular Structure 2020, 1202, https://doi.org/10.1016/j.molstruc.2019.127296.

45. Rahdar, A.; Beyzaei, H.; Askari, F.; Kyzas, G.Z. Gum-based cerium oxide nanoparticles for antimicrobial assay. Applied Physics A 2020, 126, 1-9, https://doi.org/10.1007/s00339-020-03507-4.

46. Davarpanah, A.M.; Rahdar, A.; Dastnae, M.A.; Zeybek, O.; Beyzaei, H. (1-x)BaFe12O19/ xCoFe2O4 hard/soft magnetic nanocomposites: Synthesis, physical characterization, and antibacterial activities study. Journal of Molecular Structure 2019, 1175, 445-449, https://doi.org/10.1016/j.molstruc.2018.07.092.

47. Rahdar, A.; Hajinezhad, M.R.; Sargazi, S.; Barani, M.; Bilal, M.; Kyzas, G.Z. Deferasirox-loaded pluronic nanomicelles: Synthesis, characterization, in vitro and in vivo studies. Journal of Molecular Liquids 2020, https://doi.org/10.1016/j.molliq.2020.114605.

48. Souto, E.B.; Fernandes, A.R.; Martins-Gomes, C.; Coutinho, T.E.; Durazzo, A.; Lucarini, M.; Souto, S.B.; Silva, A.M.; Santini, A. Nanomaterials for Skin Delivery of Cosmeceuticals and Pharmaceuticals. Applied Sciences 2020, 10, https://doi.org/10.3390/app10051594.

49. Carter, P.; Narasimhan, B.; Wang, Q. Biocompatible nanoparticles and vesicular systems in transdermal drug delivery for various skin diseases. International Journal of Pharmaceutics 2019, 555, 49-62, https://doi.org/10.1016/j.ijpharm.2018.11.032.

50. Severino, P.; Fangueiro, J.F.; Ferreira, S.V.; Basso, R.; Chaud, M.V.; Santana, M.H.A.; Rosmaninho, A.; Souto, E.B. Nanoemulsions and nanoparticles for non-melanoma skin cancer: effects of lipid materials. Clinical and Translational Oncology 2013, 15, 417-424, https://doi.org/10.1007/s12094-012-0982-0.

51. Dianzani, C.; Zara, G.P.; Maina, G.; Pettazzoni, P.; Pizzimenti, S.; Rossi, F.; Gigliotti, C.L.; Ciamporcero, E.S.; Daga, M.; Barrera, G. Drug Delivery Nanoparticles in Skin Cancers. BioMed Research International 2014, 2014, https://doi.org/10.1155/2014/895986.

52. Papakostas, D.; Rancan, F.; Sterry, W.; Blume-Peytavi, U.; Vogt, A. Nanoparticles in dermatology. Archives of Dermatological Research 2011, 303, https://doi.org/10.1007/s00403-011-1163-7.

53. Huber, L.A.; Pereira, T.A.; Ramos, D.N.; Rezende, L.C.; Emery, F.S.; Sobral, L.M.; Leopoldino, A.M.; Lopez, R.F. Topical Skin Cancer Therapy Using Doxorubicin-Loaded Cationic Lipid Nanoparticles and lontophoresis. J Biomed Nanotechnol 2015, 11, 1975-1988, https://doi.org/10.1166/jbn.2015.2139.

54. Amasya, G.; Aksu, B.; Badilli, U.; Onay-Besikci, A.; Tarimci, N. QbD guided early pharmaceutical development study: Production of lipid nanoparticles by high pressure homogenization for skin cancer treatment. International Journal of Pharmaceutics 2019, 563, 110-121, https://doi.org/10.1016/j.ijpharm.2019.03.056.

55. Jain, B.; Singh, B.; Katare, O.P.; Vyas, S.P. Development and characterization of minoxidil-loaded liposomal system for delivery to pilosebaceous units. Journal of Liposome Research 2010, 20, 105-114, https://doi.org/10.3109/08982100903161449.

56. Teskač, K.; Kristl, J. The evidence for solid lipid nanoparticles mediated cell uptake of resveratrol. International Journal of Pharmaceutics 2010, 390, 61-69, https://doi.org/10.1016/j.ijpharm.2009.10.011.

57. Jose, A.; Labala, S.; Ninave, K.M.; Gade, S.K.; Venuganti, V.V.K. Effective Skin Cancer Treatment by Topical Co-delivery of Curcumin and STAT3 siRNA Using Cationic Liposomes. AAPS PharmSciTech 2018, 19, 166-175, https://doi.org/10.1208/s12249-017-0833-y.

58. Caddeo, C.; Nacher, A.; Vassallo, A.; Armentano, M.F.; Pons, R.; Fernàndez-Busquets, X.; Carbone, C.; Valenti, D.; Fadda, A.M.; Manconi, M. Effect of quercetin and resveratrol co-incorporated in liposomes against inflammatory/oxidative response associated with skin cancer. Int J Pharm 2016, 513, 153-163, https://doi.org/10.1016/j.ijpharm.2016.09.014.

59. Petrilli, R.; Eloy, J.O.; Saggioro, F.P.; Chesca, D.L.; de Souza, M.C.; Dias, M.V.S.; daSilva, L.L.P.; Lee, R.J.; Lopez, R.F.V. Skin cancer treatment effectiveness is improved by iontophoresis of EGFR-targeted liposomes containing 5-FU compared with subcutaneous injection. Journal of Controlled Release 2018, 283, 151-162, https://doi.org/10.1016/j.jconrel.2018.05.038. 
60. Zhang, Z.; Tsai, P.C.; Ramezanli, T.; Michniak-Kohn, B.B. Polymeric nanoparticles-based topical delivery systems for the treatment of dermatological diseases. Wiley interdisciplinary reviews. Nanomedicine and nanobiotechnology 2013, 5, 205-218.

61. Marchiori, M.L.; Lubini, G.; Dalla Nora, G.; Friedrich, R.B.; Fontana, M.C.; Ourique, A.F.; Bastos, M.O.; Rigo, L.A.; Silva, C.B.; Tedesco, S.B.; Beck, R.C.R. Hydrogel containing dexamethasone-loaded nanocapsules for cutaneous administration: preparation, characterization, and in vitro drug release study. Drug Development and Industrial Pharmacy 2010, 36, 962-971, https://doi.org/10.3109/03639041003598960.

62. Guterres, S.S.; Alves, M.P.; Pohlmann, A.R. Polymeric nanoparticles, nanospheres and nanocapsules, for cutaneous applications. Drug Target Insights 2007, 2, 147-157.

63. Tan, Q.; Liu, W.; Guo, C.; Zhai, G. Preparation and evaluation of quercetin-loaded lecithin-chitosan nanoparticles for topical delivery. Int $J$ Nanomedicine 2011, 6, 1621-1630, https://doi.org/10.2147/ijn.S22411.

64. Nair, R.S.; Morris, A.; Billa, N.; Leong, C.O. An Evaluation of Curcumin-Encapsulated Chitosan Nanoparticles for Transdermal Delivery. AAPS PharmSciTech 2019, 20, https://doi.org/10.1208/s12249018-1279-6.

65. Özbaş-Turan, S.; Akbuğa, J.; Sezer, A.D. Topical Application of Antisense Oligonucleotide-Loaded Chitosan Nanoparticles to Rats. Oligonucleotides 2010, 20, 147-153, https://doi.org/10.1089/oli.2009.0222.

66. Dias, M.F. Desenvolvimento e avaliação da atividade quimiopreventiva de nanopartículas contendo imiquimode em modelo murino de câncer de pele. 2018.

67. Gamal-Eldeen, A.M.; El-Daly, S.M.; Borai, I.H.; Wafay, H.A.; Abdel-Ghaffar, A.-R.B. Photodynamic therapeutic effect of indocyanine green entrapped in polymeric nanoparticles and their anti-EGFR-conjugate in skin cancer in CD1 mice. Photodiagnosis and Photodynamic Therapy 2013, 10, 446-459, https://doi.org/10.1016/j.pdpdt.2013.03.013.

68. Pandey, M.; Choudhury, H.; Gunasegaran, T.A.P.; Nathan, S.S.; Md, S.; Gorain, B.; Tripathy, M.; Hussain, Z. Hyaluronic acid-modified betamethasone encapsulated polymeric nanoparticles: fabrication, characterisation, in vitro release kinetics, and dermal targeting. Drug Deliv Transl Res 2019, 9, 520-533, https://doi.org/10.1007/s13346-018-0480-1.

69. Bhatnagar, P.; Pant, A.B.; Shukla, Y.; Chaudhari, B.; Kumar, P.; Gupta, K.C. Bromelain nanoparticles protect against 7,12-dimethylbenz[a]anthracene induced skin carcinogenesis in mouse model. European journal of pharmaceutics and biopharmaceutics: official journal of Arbeitsgemeinschaft fur Pharmazeutische Verfahrenstechnik e.V 2015, 91, 35-46, https://doi.org/10.1016/j.ejpb.2015.01.015.

70. Bayat, S.; Amiri, N.; Pishavar, E.; Kalalinia, F.; Movaffagh, J.; Hashemi, M. Bromelain-loaded chitosan nanofibers prepared by electrospinning method for burn wound healing in animal models. Life Sciences 2019, 229, 57-66, https://doi.org/10.1016/j.lfs.2019.05.028.

71. Primo, F.L.; Rodrigues, M.M.A.; Simioni, A.R.; Bentley, M.V.L.B.; Morais, P.C.; Tedesco, A.C. In vitro studies of cutaneous retention of magnetic nanoemulsion loaded with zinc phthalocyanine for synergic use in skin cancer treatment. Journal of Magnetism and Magnetic Materials 2008, 320, e211-e214, https://doi.org/10.1016/j.jmmm.2008.02.050.

72. Primo, F.L.; Michieleto, L.; Rodrigues, M.A.M.; Macaroff, P.P.; Morais, P.C.; Lacava, Z.G.M.; Bentley, M.V.L.B.; Tedesco, A.C. Magnetic nanoemulsions as drug delivery system for Foscan®: Skin permeation and retention in vitro assays for topical application in photodynamic therapy (PDT) of skin cancer. Journal of Magnetism and Magnetic Materials 2007, 311, 354-357, https://doi.org/10.1016/j.jmmm.2006.10.1183.

73. Giacone, D.V.; Dartora, V.F.M.C.; de Matos, J.K.R.; Passos, J.S.; Miranda, D.A.G.; de Oliveira, E.A.; Silveira, E.R.; Costa-Lotufo, L.V.; Maria-Engler, S.S.; Lopes, L.B. Effect of nanoemulsion modification with chitosan and sodium alginate on the topical delivery and efficacy of the cytotoxic agent piplartine in 2D and 3D skin cancer models. International Journal of Biological Macromolecules 2020, 165, 1055-1065, https://doi.org/10.1016/j.ijbiomac.2020.09.167.

74. Falamas, A.; Dehelean, C.A.; Cinta Pinzaru, S. Monitoring of betulin nanoemulsion treatment and molecular changes in mouse skin cancer using surface enhanced Raman spectroscopy. Vibrational Spectroscopy 2018, 95, 44-50, https://doi.org/10.1016/j.vibspec.2018.01.004.

75. Yousef, S.A.; Mohammed, Y.H.; Namjoshi, S.; Grice, J.E.; Benson, H.A.E.; Sakran, W.; Roberts, M.S. Mechanistic Evaluation of Enhanced Curcumin Delivery through Human Skin In Vitro from Optimised Nanoemulsion Formulations Fabricated with Different Penetration Enhancers. Pharmaceutics 2019, 11, https://doi.org/10.3390/pharmaceutics11120639.

76. Loh, K.J.; Hou, T.-C.; Lynch, J.P.; Kotov, N.A. Carbon Nanotube Sensing Skins for Spatial Strain and Impact Damage Identification. Journal of Nondestructive Evaluation 2009, 28, 9-25, https://doi.org/10.1007/s10921-009-0043-y.

77. Shvedova, A.A.; Castranova, V.; Kisin, E.R.; Schwegler-Berry, D.; Murray, A.R.; Gandelsman, V.Z.; Maynard, A.; Baron, P. Exposure to carbon nanotube material: assessment of nanotube cytotoxicity using human keratinocyte cells. Journal of toxicology and environmental health. Part A 2003, 66, 1909-1926, https://doi.org/10.1080/713853956. 
78. Ding, L.; Stilwell, J.; Zhang, T.; Elboudwarej, O.; Jiang, H.; Selegue, J.P.; Cooke, P.A.; Gray, J.W.; Chen, F.F. Molecular characterization of the cytotoxic mechanism of multiwall carbon nanotubes and nano-onions on human skin fibroblast. Nano Lett 2005, 5, 2448-2464, https://doi.org/10.1021/n1051748o.

79. Hesabi, M.; Hesabi, M. The interaction between carbon nanotube and skin anti-cancer drugs: a DFT and NBO approach. Journal of Nanostructure in Chemistry 2013, 3, https://doi.org/10.1186/2193-8865-3-22.

80. Moon, H.K.; Lee, S.H.; Choi, H.C. In Vivo Near-Infrared Mediated Tumor Destruction by Photothermal Effect of Carbon Nanotubes. ACS Nano 2009, 3, 3707-3713, https://doi.org/10.1021/nn900904h.

81. Sahoo, N.G.; Rana, S.; Cho, J.W.; Li, L.; Chan, S.H. Polymer nanocomposites based on functionalized carbon nanotubes. Progress in Polymer Science 2010, 35, 837-867, https://doi.org/10.1016/j.progpolymsci.2010.03.002.

82. Kahraman, E.; Neşetoğlu, N.; Güngör, S.; Ünal, D.Ş.; Özsoy, Y. The combination of nanomicelles with terpenes for enhancement of skin drug delivery. International Journal of Pharmaceutics 2018, 551, 133140, https://doi.org/10.1016/j.ijpharm.2018.08.053.

83. Vilsinski, B.H.; Witt, M.A.; Barbosa, P.M.; Montanha, M.C.; Nunes, C.S.; Bellettini, I.C.; de Castro, L.V.; Sato, F.; Baesso, M.L.; Muniz, E.C.; Caetano, W. Formulation of chloroaluminum phthalocyanine incorporated into PS-b-PAA diblock copolymer nanomicelles. Journal of Molecular Liquids 2018, 271, 949958, https://doi.org/10.1016/j.molliq.2018.09.034.

84. Wang, M.-Z.; Niu, J.; Ma, H.-J.; Dad, H.A.; Shao, H.-T.; Yuan, T.-J.; Peng, L.-H. Transdermal siRNA delivery by $\mathrm{pH}$-switchable micelles with targeting effect suppress skin melanoma progression. Journal of Controlled Release 2020, 322, 95-107, https://doi.org/10.1016/j.jconrel.2020.03.023.

85. Gong, C.Y.; Shi, S.; Dong, P.W.; Yang, B.; Qi, X.R.; Guo, G.; Gu, Y.C.; Zhao, X.; Wei, Y.Q.; Qian, Z.Y. Biodegradable in situ gel-forming controlled drug delivery system based on thermosensitive PCL-PEG-PCL hydrogel: part 1--Synthesis, characterization, and acute toxicity evaluation. J Pharm Sci 2009, 98, 46844694, https://doi.org/10.1002/jps.21780.

86. Xu, H.; Wen, Y.; Chen, S.; Zhu, L.; Feng, R.; Song, Z. Paclitaxel skin delivery by micelles-embedded Carbopol 940 hydrogel for local therapy of melanoma. International Journal of Pharmaceutics 2020, 587, https://doi.org/10.1016/j.ijpharm.2020.119626.

87. Rosa, J.; Suzuki, I.; Kravicz, M.; Caron, A.; Pupo, A.V.; Praça, F.G.; Bentley, M. Current Non-viral siRNA Delivery Systems as a Promising Treatment of Skin Diseases. Curr Pharm Des 2018, 24, 2644-2663, https://doi.org/10.2174/1381612824666180807120017.

88. Xue, B.; Wang, Y.; Tang, X.; Xie, P.; Wang, Y.; Luo, F.; Wu, C.; Qian, Z. Biodegradable self-assembled MPEG-PCL micelles for hydrophobic oridonin delivery in vitro. J Biomed Nanotechnol 2012, 8, 80-89, https://doi.org/10.1166/jbn.2012.1358.

89. Jose, A.; Labala, S.; Venuganti, V.V. Co-delivery of curcumin and STAT3 siRNA using deformable cationic liposomes to treat skin cancer. J Drug Target 2017, 25, 330-341, https://doi.org/10.1080/1061186x.2016.1258567.

90. Pornpattananangkul, D.; Olson, S.; Aryal, S.; Sartor, M.; Huang, C.-M.; Vecchio, K.; Zhang, L. StimuliResponsive Liposome Fusion Mediated by Gold Nanoparticles. ACS Nano 2010, 4, 1935-1942, https://doi.org/10.1021/nn9018587.

91. Limón, D.; Fábrega, M.J.; Calpena, A.C.; Badia, J.; Baldomà, L.; Pérez-García, L. Multifunctional Serine Protease Inhibitor-Coated Water-Soluble Gold Nanoparticles as a Novel Targeted Approach for the Treatment of Inflammatory Skin Diseases. Bioconjugate Chemistry 2018, 29, 1060-1072, https://doi.org/10.1021/acs.bioconjchem.7b00717.

92. Preet, S.; Pandey, S.K.; Kaur, K.; Chauhan, S.; Saini, A. Gold nanoparticles assisted co-delivery of nisin and doxorubicin against murine skin cancer. Journal of Drug Delivery Science and Technology 2019, 53, https://doi.org/10.1016/j.jddst.2019.101147.

93. Nirmala, J.G.; Akila, S.; Nadar, M.S.A.M.; Narendhirakannan, R.T.; Chatterjee, S. Biosynthesized Vitis vinifera seed gold nanoparticles induce apoptotic cell death in A431 skin cancer cells. RSC Advances 2016, 6, 82205-82218, https://doi.org/10.1039/C6RA16310F.

94. Fratoddi, I.; Benassi, L.; Botti, E.; Vaschieri, C.; Venditti, I.; Bessar, H.; Samir, M.A.; Azzoni, P.; Magnoni, C.; Costanzo, A.; Casagrande, V.; Federici, M.; Bianchi, L.; Pellacani, G. Effects of topical methotrexate loaded gold nanoparticle in cutaneous inflammatory mouse model. Nanomedicine: Nanotechnology, Biology and Medicine 2019, 17, 276-286, https://doi.org/10.1016/j.nano.2019.01.006.

95. Li, Z.; Barnes, J.C.; Bosoy, A.; Stoddart, J.F.; Zink, J.I. Mesoporous silica nanoparticles in biomedical applications. Chemical Society Reviews 2012, 41, 2590-2605, https://doi.org/10.1039/C1CS15246G.

96. Hirai, T.; Yoshikawa, T.; Nabeshi, H.; Yoshida, T.; Tochigi, S.; Ichihashi, K.; Uji, M.; Akase, T.; Nagano, K.; Abe, Y.; Kamada, H.; Itoh, N.; Tsunoda, S.; Yoshioka, Y.; Tsutsumi, Y. Amorphous silica nanoparticles size-dependently aggravate atopic dermatitis-like skin lesions following an intradermal injection. Part Fibre Toxicol 2012, 9, https://doi.org/10.1186/1743-8977-9-3.

97. Pan, J.; Ruan, W.; Qin, M.; Long, Y.; Wan, T.; Yu, K.; Zhai, Y.; Wu, C.; Xu, Y. Intradermal delivery of STAT3 siRNA to treat melanoma via dissolving microneedles. Scientific Reports 2018, 8, 1-11, https://doi.org/10.1038/s41598-018-19463-2. 
98. Ma, X.; Teh, C.; Zhang, Q.; Borah, P.; Choong, C.; Korzh, V.; Zhao, Y. Redox-responsive mesoporous silica nanoparticles: a physiologically sensitive codelivery vehicle for siRNA and doxorubicin. Antioxidants \& redox signaling 2014, 21, 707-722, https://doi.org/10.1089/ars.2012.5076.

99. Li, F.; Jin, L.; He, L.; Deng, Y.; He, N. Nanoparticles Applied for Therapy and Diagnosis in Common Diseases. Science of Advanced Materials 2015, 7, 2103-2122, https://doi.org/10.1166/sam.2015.1982.

100. Rosenholm, J.M.; Mamaeva, V.; Sahlgren, C.; Lindén, M. Nanoparticles in targeted cancer therapy: mesoporous silica nanoparticles entering preclinical development stage. Nanomedicine (London, England) 2012, 7, 111-120, https://doi.org/10.2217/nnm.11.166.

101. Rancan, F.; Gao, Q.; Graf, C.; Troppens, S.; Hadam, S.; Hackbarth, S.; Kembuan, C.; Blume-Peytavi, U.; Rühl, E.; Lademann, J.; Vogt, A. Skin penetration and cellular uptake of amorphous silica nanoparticles with variable size, surface functionalization, and colloidal stability. ACS Nano 2012, 6, 6829-6842, https://doi.org/10.1021/nn301622h.

102. Gonçalves, L.C.; Seabra, A.B.; Pelegrino, M.T.; de Araujo, D.R.; Bernardes, J.S.; Haddad, P.S. Superparamagnetic iron oxide nanoparticles dispersed in Pluronic F127 hydrogel: potential uses in topical applications. RSC Advances 2017, 7, 14496-14503, https://doi.org/10.1039/C6RA28633J.

103. Rao, Y.F.; Chen, W.; Liang, X.G.; Huang, Y.Z.; Miao, J.; Liu, L.; Lou, Y.; Zhang, X.G.; Wang, B.; Tang, R.K.; Chen, Z.; Lu, X.Y. Epirubicin-loaded superparamagnetic iron-oxide nanoparticles for transdermal delivery: cancer therapy by circumventing the skin barrier. Small 2015, 11, 239-247.

104. Cengelli, F.; Voinesco, F.; Juillerat-Jeanneret, L. Interaction of cationic ultrasmall superparamagnetic iron oxide nanoparticles with human melanoma cells. Nanomedicine (London, England) 2010, 5, 1075-1087, https://doi.org/10.2217/nnm.10.79.

105. Choi, G.H.; Seo, S.J.; Kim, K.H.; Kim, H.T.; Park, S.H.; Lim, J.H.; Kim, J.K. Photon activated therapy (PAT) using monochromatic synchrotron X-rays and iron oxide nanoparticles in a mouse tumor model: feasibility study of PAT for the treatment of superficial malignancy. Radiation oncology (London, England) 2012, 7, https://doi.org/10.1186/1748-717x-7-184.

106. Musazzi, U.M.; Cencetti, C.; Franzé, S.; Zoratto, N.; Di Meo, C.; Procacci, P.; Matricardi, P.; Cilurzo, F. Gellan Nanohydrogels: Novel Nanodelivery Systems for Cutaneous Administration of Piroxicam. Molecular Pharmaceutics 2018, 15, 1028-1036, https://doi.org/10.1021/acs.molpharmaceut.7b00926.

107. Mogensen, M.; Jemec, G.B. Diagnosis of nonmelanoma skin cancer/keratinocyte carcinoma: a review of diagnostic accuracy of nonmelanoma skin cancer diagnostic tests and technologies. Dermatol Surg 2007, 33, 1158-1174.

108. Grossman, D.C.; Curry, S.J.; Owens, D.K.; Barry, M.J.; Caughey, A.B.; Davidson, K.W.; Doubeni, C.A.; Epling, J.W.Jr.; Kemper, A.R.; Krist, A.H.; Kubik, M.; Landefeld, S.; Mangione, C.M.; Silverstein, M.; Simon, M.A.; Tseng, C.W. Behavioral Counseling to Prevent Skin Cancer: US Preventive Services Task Force Recommendation Statement. Jama 2018, 319, 1134-1142, https://doi.org/10.1001/jama.2018.1623.

109. Qadir, M. I. Skin cancer: Etiology and management. Pakistan Journal of Pharmaceutical Sciences $2016,29$.

110. Naves, L.B.; Dhand, C.; Venugopal, J.R.; Rajamani, L.; Ramakrishna, S.; Almeida, L. Nanotechnology for the treatment of melanoma skin cancer. Progress in biomaterials 2017, 6, 13-26, https://doi.org/10.1007/s40204-017-0064-z.

111. Peng, G.; Hakim, M.; Broza, Y.Y.; Billan, S.; Abdah-Bortnyak, R.; Kuten, A.; Tisch, U.; Haick, H. Detection of lung, breast, colorectal, and prostate cancers from exhaled breath using a single array of nanosensors. British Journal of Cancer 2010, 103, 542-551, https://doi.org/10.1038/sj.bjc.6605810.

112. Rodríguez, D.; Morrison, C.J.; Overall, C.M. Matrix metalloproteinases: What do they not do? New substrates and biological roles identified by murine models and proteomics. Biochimica et Biophysica Acta (BBA) - Molecular Cell Research 2010, 1803, 39-54, https://doi.org/10.1016/j.bbamcr.2009.09.015.

113. Zhang, Z.; Zhu, S.; Yang, Y.; Ma, X.; Guo, S. Matrix metalloproteinase-12 expression is increased in cutaneous melanoma and associated with tumor aggressiveness. Tumour biology: the journal of the International Society for Oncodevelopmental Biology and Medicine 2015, 36, 8593-8600, https://doi.org/10.1007/s13277-015-3622-9.

114. Lin, T.E.; Bondarenko, A.; Lesch, A.; Pick, H.; Cortés-Salazar, F.; Girault, H.H. Monitoring Tyrosinase Expression in Non-metastatic and Metastatic Melanoma Tissues by Scanning Electrochemical Microscopy. Angewandte Chemie (International ed. in English) 2016, 55, 3813-3816.

115. Tondera, C.; Ullm, S.; Laube, M.; Meister, S.; Neuber, C.; Mosch, B.; Kniess, T.; Pietzsch, J. Optical imaging of COX-2: studies on an autofluorescent 2,3-diaryl-substituted indole-based cyclooxygenase-2 inhibitor. Biochem Biophys Res Commun 2015, 458, 40-45, https://doi.org/10.1016/j.bbrc.2015.01.057.

116. Kim, S.-H.; Hashimoto, Y.; Cho, S.-N.; Roszik, J.; Milton, D.R.; Dal, F.; Kim, S.F.; Menter, D.G.; Yang, P.; Ekmekcioglu, S.; Grimm, E.A. Microsomal PGE2 synthase-1 regulates melanoma cell survival and associates with melanoma disease progression. Pigment Cell Melanoma Res 2016, 29, 297-308, https://doi.org/10.1111/pcmr.12455.

117. Lugowska, I.; Kowalska, M.; Fuksiewicz, M.; Kotowicz, B.; Mierzejewska, E.; Koseła-Paterczyk, H.; Szamotulska, K.; Rutkowski, P. Serum markers in early-stage and locally advanced melanoma. Tumour biology : the journal of the International Society for Oncodevelopmental Biology and Medicine 2015, 36, 8277-8285, https://doi.org/10.1007/s13277-015-3564-2. 
118. Hagerty, J.R.; Stanley, R.J.; Almubarak, H.A.; Lama, N.; Kasmi, R.; Guo, P.; Drugge, R.J.; Rabinovitz, H.S.; Oliviero, M.; Stoecker, W.V. Deep Learning and Handcrafted Method Fusion: Higher Diagnostic Accuracy for Melanoma Dermoscopy Images. IEEE journal of biomedical and health informatics 2019, 23, 13851391, https://doi.org/10.1109/jbhi.2019.2891049.

119. Gebhardt, C.; Sevko, A.; Jiang, H.; Lichtenberger, R.; Reith, M.; Tarnanidis, K.; Holland-Letz, T.; Umansky, L.; Beckhove, P.; Sucker, A.; Schadendorf, D.; Utikal, J.; Umansky, V. Myeloid Cells and Related Chronic Inflammatory Factors as Novel Predictive Markers in Melanoma Treatment with Ipilimumab. Clinical cancer research : an official journal of the American Association for Cancer Research 2015, 21, 5453-5459, https://doi.org/10.1158/1078-0432.Ccr-15-0676.

120. Warton, K.; Mahon, K.L.; Samimi, G. Methylated circulating tumor DNA in blood: power in cancer prognosis and response. Endocrine-related cancer 2016, 23, R157-171, https://doi.org/10.1530/erc-15-0369.

121. Yoshioka, Y.; Kosaka, N.; Konishi, Y.; Ohta, H.; Okamoto, H.; Sonoda, H.; Nonaka, R.; Yamamoto, H.; Ishii, H.; Mori, M.; Furuta, K.; Nakajima, T.; Hayashi, H.; Sugisaki, H.; Higashimoto, H.; Kato, T.; Takeshita, F.; Ochiya, T. Ultra-sensitive liquid biopsy of circulating extracellular vesicles using ExoScreen. Nature Communications 2014, 5, 1-8, https://doi.org/10.1038/ncomms4591.

122. Amirjahan, M.; Sujatha, D.N. Comparative analysis of various classification algorithms for skin Cancer detection. PG \& Research Department of Computer Science, Raja Doraisingam Govt. Art College, Sivagangai, Tamil Nadu, India 2016, 199-205.

123. Coroiu, A.; Moran, C.; Bergeron, C.; Thombs, B.D.; Geller, A.C.; Kingsland, E.; Körner, A. Operationalization of skin self-examination in randomized controlled trials with individuals at increased risk for melanoma: A systematic review. Patient education and counseling 2020, 103, 1013-1026, https://doi.org/10.1016/j.pec.2019.12.009.

124. Lallas, A.; Longo, C.; Manfredini, M.; Benati, E.; Babino, G.; Chinazzo, C.; Apalla, Z.; Papageorgiou, C.; Moscarella, E.; Kyrgidis, A.; Argenziano, G. Accuracy of Dermoscopic Criteria for the Diagnosis of Melanoma In Situ. JAMA Dermatol 2018, 154, 414-419, https://doi.org/10.1001/jamadermatol.2017.6447.

125. Argenziano, G.; Catricalà, C.; Ardigo, M.; Buccini, P.; De Simone, P.; Eibenschutz, L.; Ferrari, A.; Mariani, G.; Silipo, V.; Sperduti, I.; Zalaudek, I. Seven-point checklist of dermoscopy revisited. The British journal of dermatology 2011, 164, 785-790, https://doi.org/10.1111/j.1365-2133.2010.10194.x.

126. Shobatake, C.; Miyagawa, F.; Ogawa, K.; Azukizawa, H.; Hirai, T.; Kuwahara, M.; Asada, H. Preoperative assessment of tumour thickness and vascularity using high-frequency ultrasonography in ten cases of cutaneous melanoma. European journal of dermatology: EJD 2018, 28, 256-258, https://doi.org/10.1684/ejd.2018.3223.

127. Russell, C.; Ward, A.C.; Vezza, V.; Hoskisson, P.; Alcorn, D.; Steenson, D.P.; Corrigan, D.K. Development of a needle shaped microelectrode for electrochemical detection of the sepsis biomarker interleukin-6 (IL-6) in real time. Biosensors and Bioelectronics 2019, 126, 806-814, https://doi.org/10.1016/j.bios.2018.11.053.

128. Eftekhari, A.; Ahmadian, E.; Salatin, S.; Sharifi, S.; Dizaj, S.M.; Khalilov, R.; Hasanzadeh, M. Current analytical approaches in diagnosis of melanoma. TrAC Trends in Analytical Chemistry 2019, 116, 122-135, https://doi.org/10.1016/j.trac.2019.05.004.

129. Felix, F.S.; Angnes, L. Electrochemical immunosensors - A powerful tool for analytical applications. Biosensors and Bioelectronics 2018, 102, 470-478, https://doi.org/10.1016/j.bios.2017.11.029.

130. Wen, W.; Yan, X.; Zhu, C.; Du, D.; Lin, Y. Recent Advances in Electrochemical Immunosensors. Analytical Chemistry 2017, 89, 138-156, https://doi.org/10.1021/acs.analchem.6b04281.

131. Seenivasan, R.; Maddodi, N.; Setaluri, V.; Gunasekaran, S. An electrochemical immunosensing method for detecting melanoma cells. Biosensors \& bioelectronics 2015, 68, 508-515, https://doi.org/10.1016/j.bios.2015.01.022.

132. Ren, X.; Yan, T.; Zhang, Y.; Wu, D.; Ma, H.; Li, H.; Du, B.; Wei, Q. Nanosheet Au/Co3O4-based ultrasensitive nonenzymatic immunosensor for melanoma adhesion molecule antigen. Biosensors and Bioelectronics 2014, 58, 345-350, https://doi.org/10.1016/j.bios.2014.03.010.

133. Heikenfeld, J.; Jajack, A.; Rogers, J.; Gutruf, P.; Tian, L.; Pan, T.; Li, R.; Khine, M.; Kim, J.; Wang, J.; Kim, J. Wearable sensors: modalities, challenges, and prospects. Lab on a Chip 2018, 18, 217-248, https://doi.org/10.1039/C7LC00914C.

134. Ciui, B.; Martin, A.; Mishra, R.K.; Brunetti, B.; Nakagawa, T.; Dawkins, T.J.; Lyu, M.; Cristea, C.; Sandulescu, R.; Wang, J. Wearable Wireless Tyrosinase Bandage and Microneedle Sensors: Toward Melanoma Screening. Adv Healthc Mater 2018, 7.

135. Bertok, T.; Lorencova, L.; Chocholova, E.; Jane, E.; Vikartovska, A.; Kasak, P.; Tkac, J. Electrochemical Impedance Spectroscopy Based Biosensors: Mechanistic Principles, Analytical Examples and Challenges towards Commercialization for Assays of Protein Cancer Biomarkers. ChemElectroChem 2019, 6, 989-1003, https://doi.org/10.1002/celc.201800848.

136. Braun, R.P.; Mangana, J.; Goldinger, S.; French, L.; Dummer, R.; Marghoob, A.A. Electrical Impedance Spectroscopy in Skin Cancer Diagnosis. Dermatologic clinics 2017, 35, 489-493, https://doi.org/10.1016/j.det.2017.06.009. 
137. Prathap, M.U.A.; Rodríguez, C.I.; Sadak, O.; Guan, J.; Setaluri, V.; Gunasekaran, S. Ultrasensitive electrochemical immunoassay for melanoma cells using mesoporous polyaniline. Chemical Communications 2018, 54, 710-714, https://doi.org/10.1039/C7CC09248B.

138. Yen, L.-C.; Pan, T.-M.; Lee, C.-H.; Chao, T.-S. Label-free and real-time detection of ferritin using a hornlike polycrystalline-silicon nanowire field-effect transistor biosensor. Sensors and Actuators B: Chemical 2016, 230, 398-404, https://doi.org/10.1016/j.snb.2016.02.095.

139. Maedler, C.; Kim, D.; Spanjaard, R.A.; Hong, M.; Erramilli, S.; Mohanty, P. Sensing of the Melanoma Biomarker TROY Using Silicon Nanowire Field-Effect Transistors. ACS Sensors 2016, 1, 696-701, https://doi.org/10.1021/acssensors.6b00017.

140. Eivazzadeh-Keihan, R.; Pashazadeh-Panahi, P.; Mahmoudi, T.; Chenab, K.K.; Baradaran, B.; Hashemzaei, M.; Radinekiyan, F.; Mokhtarzadeh, A.; Maleki, A. Dengue virus: a review on advances in detection and trends - from conventional methods to novel biosensors. Microchimica Acta 2019, 186, https://doi.org/10.1007/s00604-019-3420-y.

141. Li, Z.; wang, y.; Zhang, X.; Zeng, C.; Hu, L.; Liang, X.-J. A Tyrosinase-triggered Oxidative Reaction-Based "Turn-on" Fluorescent Probe for Imaging in Living Melanoma Cells. Sensors and Actuators B: Chemical 2016, 242, https://doi.org/10.1016/j.snb.2016.11.011.

142. Hu, J.-J.; Bai, X.-L.; Liu, Y.-M.; Liao, X. Functionalized carbon quantum dots with dopamine for tyrosinase activity analysis. Analytica Chimica Acta 2017, 995, 99-105, https://doi.org/10.1016/j.aca.2017.09.038.

143. Li, P.; He, H.; Wang, Z.; Feng, M.; Jin, H.; Wu, Y.; Zhang, L.; Zhang, L.; Tang, X. Sensitive Detection of Single-Nucleotide Mutation in the BRAF Mutation Site (V600E) of Human Melanoma Using PhosphatePyrene-Labeled DNA Probes. Analytical Chemistry 2016, 88, 883-889, https://doi.org/10.1021/acs.analchem.5b03523.

144. Sweeny, L.; Prince, A.; Patel, N.; Moore, L.S.; Rosenthal, E.L.; Hughley, B.B.; Warram, J.M. Antiangiogenic antibody improves melanoma detection by fluorescently labeled therapeutic antibodies. The Laryngoscope 2016, 126, E387-E395, https://doi.org/10.1002/lary.26215.

145. Tu, W.; Wang, Z.; Dai, Z. Selective photoelectrochemical architectures for biosensing: Design, mechanism and responsibility. TrAC Trends in Analytical Chemistry 2018, 105, 470-483, https://doi.org/10.1016/j.trac.2018.06.007.

146. Xiong, E.; Yan, X.; Zhang, X.; Li, Y.; Yang, R.; Meng, L.; Chen, J. A new photoelectrochemical biosensor for ultrasensitive determination of nucleic acids based on a three-stage cascade signal amplification strategy. Analyst 2018, 143, 2799-2806, https://doi.org/10.1039/C8AN00609A.

147. Lopes, J.; Coelho, J.M.P.; Vieira, P.M.C.; Viana, A.S.; Gaspar, M.M.; Reis, C. Preliminary Assays towards Melanoma Cells Using Phototherapy with Gold-Based Nanomaterials. Nanomaterials (Basel) 2020, 10, https://doi.org/10.3390/nano10081536. 